\title{
Effects of Vitamin E-Coated versus Conventional Membranes in Chronic Hemodialysis Patients: A Systematic Review and Meta-Analysis
}

\author{
Graziella D'Arrigo ${ }^{a}$ Rossella Baggetta ${ }^{a}$ Giovanni Tripepi $^{a}$ Francesco Galli $^{b}$ \\ Davide Bolignano ${ }^{a}$ \\ ${ }^{a}$ CNR - Institute of Clinical Physiology, Reggio Calabria, and b ${ }^{\text {D }}$ utrition and Clinical Biochemistry Laboratory, \\ Department of Pharmaceutical Sciences, University of Perugia, Perugia, Italy
}

\section{Key Words}

Vitamin E-coated membrane - Oxidative stress .

Inflammation · Anemia $\cdot$ Hemodialysis

\begin{abstract}
Introduction: Accruing evidence suggests that vitamin Ecoated membranes (ViE-m) might improve the clinical management of chronic hemodialysis (HD) patients. Methods: We conducted a systematic review and meta-analysis of RCTs comparing ViE-m to conventional HD. Endpoints of interest were a series of biomarkers pertaining to anemia status, inflammation, oxidative stress and dialysis efficacy/status. Results: Sixty studies were included. ViE-m significantly improved the Erythropoietin Resistance Index but had no impact on other anemia parameters. As for oxidative stress and inflammation, ViE-m produced a significant decrease in interleukin- 6 levels, thiobarbituric acid reactive substances, plasma and red blood cell (RBC) malonylaldehyde and a significant increase in blood and RBC vitamin E. Conversely, ViE-m use had no impact on lipid profile, dialysis adequacy, blood pressure, albumin and uric acid. Conclusions: ViE-m might ameliorate anemia management by reducing oxidative stress and inflammation. Benefits of these bio-membranes on harder clinical outcomes are uncertain and need to be investigated by future, targeted trials.
\end{abstract}

\section{Introduction}

Improving the quality of hollow-fiber hemodialysis (HD) membranes in terms of hydraulic properties and biocompatibility has been the goal of decades of technology efforts. As a result, last-generation synthetic biomaterials have reached very high standards of performance, which when combined with the use of convective techniques have ensured better clinical outcomes of patients on maintenance HD therapy $[1,2]$.

Vitamin E has been used since the early 1990s as a blood surface modifier of cellulosic first and then synthetic hollow-fiber membranes with the aim of further improving biocompatibility and eventually providing antioxidant protection to blood cell membranes and circulating lipoproteins $[3,4]$. This is a unique example of 'bioactive' membranes with antioxidant properties that have been well characterized in vitro [5] and in vivo [6].

Previous pooled analyses demonstrated that vitamin E-coated membranes (ViE-m) may improve various surrogate parameters pertaining to oxidative stress, inflammation, anemia and nutritional status [7-9]. In particular, as ViE-m seems to ameliorate the response to erythropoiesis stimulating agents (ESAs) and to protect red

G.D. and R.B. equally contributed to the present work.

Dr. Graziella D'Arrigo, BioStat 
blood cell (RBC) by increasing RBC vitamin-E levels, it has been postulated that these membranes could be useful in daily practice for sparing ESAs in chronic HD patients $[10,11]$.

Yet, in spite of a wealth of data accruing, there is still no conclusive evidence to prove that there is a clear advantage of these membranes over standard HD therapy.

This is partly related to the poor quality of studies analyzed by previous systematic reviews, most of which were uncontrolled, pilot or cross-over trials conducted on very small populations, with questionable methodology focused on surrogate rather than patient-centered outcomes.

In previous years, new larger trials have been carried out testing the effects of ViE-m over standard HD membranes on a series of new, clinically relevant endpoints.

We therefore felt it necessary to perform a new, comprehensive systematic review and meta-analysis with the aim of summarizing the entire currently available evidence on the effects of ViE-m as compared to standard HD treatment, on any endpoint of interest including but not confined to anemia, inflammation and oxidative stress.

\section{Methods}

Data Source and Search Strategy

We performed a focused, highly sensitive literature search on Ovid-MEDLINE, PubMed and CENTRAL databases without time or language restriction up to March 2016 to identify eligible studies (online suppl. table 1; for all online suppl. material, see www. karger.com/doi/10.1159/000453444) according to PRISMA guidelines. The search was designed and performed by 3 authors (D.B., G.D. and R.B.)

\section{Study Selection and Data Extraction}

We included any randomized or nonrandomized controlled study that tested the effects of ViE-m on oxidative stress, inflammation, anemia in end-stage kidney disease patients on chronic HD treatment. Other parameters of interest, such as lipid profile, preand post-dialysis blood pressure, dialysis adequacy, serum albumin, uric acid and white blood cells (WBCs) count, were also considered. Studies were included without follow-up duration restrictions.

Studies were excluded if they dealt with the wrong intervention (e.g. vitamin E administered orally), if they did not report outcomes of interest or if they did not focus on individuals undergoing chronic dialysis treatment (e.g. HD for acute kidney injury).

Titles and abstracts were screened independently by 2 authors (G.D. and R.B.); they discarded studies that were not pertinent to the topic. Case reports, reviews, editorials, and letters were excluded from qualitative analyses but screened for potential additional references. Two authors (G.D. and R.B.) independently assessed the abstracts and the full text of these studies to determine the eligibility of these studies based on the inclusion/exclusion criteria. Additional data were eventually requested from the authors.
A third reviewer (D.B.) solved possible discrepancies on study judgments. Data extraction and analysis were performed by 2 reviewers (G.D. and R.B.) and independently verified by another (D.B.).

\section{Data Analysis}

Meta-analyses were carried out if data on the same outcome were provided by more than 2 studies.

To evaluate a possible effect of treatment on continuous variables with the same scale, we used the mean difference (MD); for variables expressed in different scales, the standardized MD (SMD) was used.

Data were pooled using the random-effects model. Data that were available as median and range were converted to mean and SD using the Hozo formula [12].

Heterogeneity was measured by the $\mathrm{chi}^{2}$ test on $\mathrm{N}-1$ degrees of freedom, with an alpha of 0.05 considered for statistical significance and the Cochrane- $\mathrm{I}^{2}[13] . \mathrm{I}^{2}$ values of $0-30,30-60$ and $>60 \%$ were assumed to correspond to low, medium and high levels of heterogeneity respectively.

When high strength of evidence for heterogeneity $\left(\mathrm{I}^{2}>30 \%\right.$ and significant $\mathrm{p}$ value from the $\mathrm{chi}^{2}$ test) was present, possible causes were explored by sensitivity and sub-group analyses based on sample size, study design, follow-up duration, age of patients and dialysis vintage.

Publication bias was investigated by the Egger's regression test and visual inspection of funnel plots for meta-analyses carried out with more than 4 studies. Statistical analyses were performed using Review Manager (RevMan; version 5.3; Copenhagen: The Nordic Cochrane Centre, The Cochrane Collaboration, 2014) and Stata/ IC (version 13.1; StataCorp LP, Tex., USA).

In order to maximize information, data on outcomes reported by single studies or data present in a descriptive way were reported narratively.

\section{Results}

\section{Search Results}

Four hundred and four potentially relevant references were initially retrieved. By screening titles and abstracts, a total of 339 citations were excluded because of search overlap, intervention not pertinent, review articles, case reports or experimental studies. Among the 85 studies selected for full text examination, 27 studies were excluded because of the following reasons: outcomes were not pertinent to the topic $(\mathrm{n}=14)$; these were uncontrolled studies on use of oral vitamin E $(n=5)$, letters, editorials, reviews or experimental studies $(n=8)$. A total of 60 articles were, therefore, reviewed in detail. Figure 1 summarizes the study flow of this review.

\section{Study Characteristics}

Main characteristics of the studies reviewed are summarized in online supplementary tables 2-5. 
Fig. 1. Study selection flow.

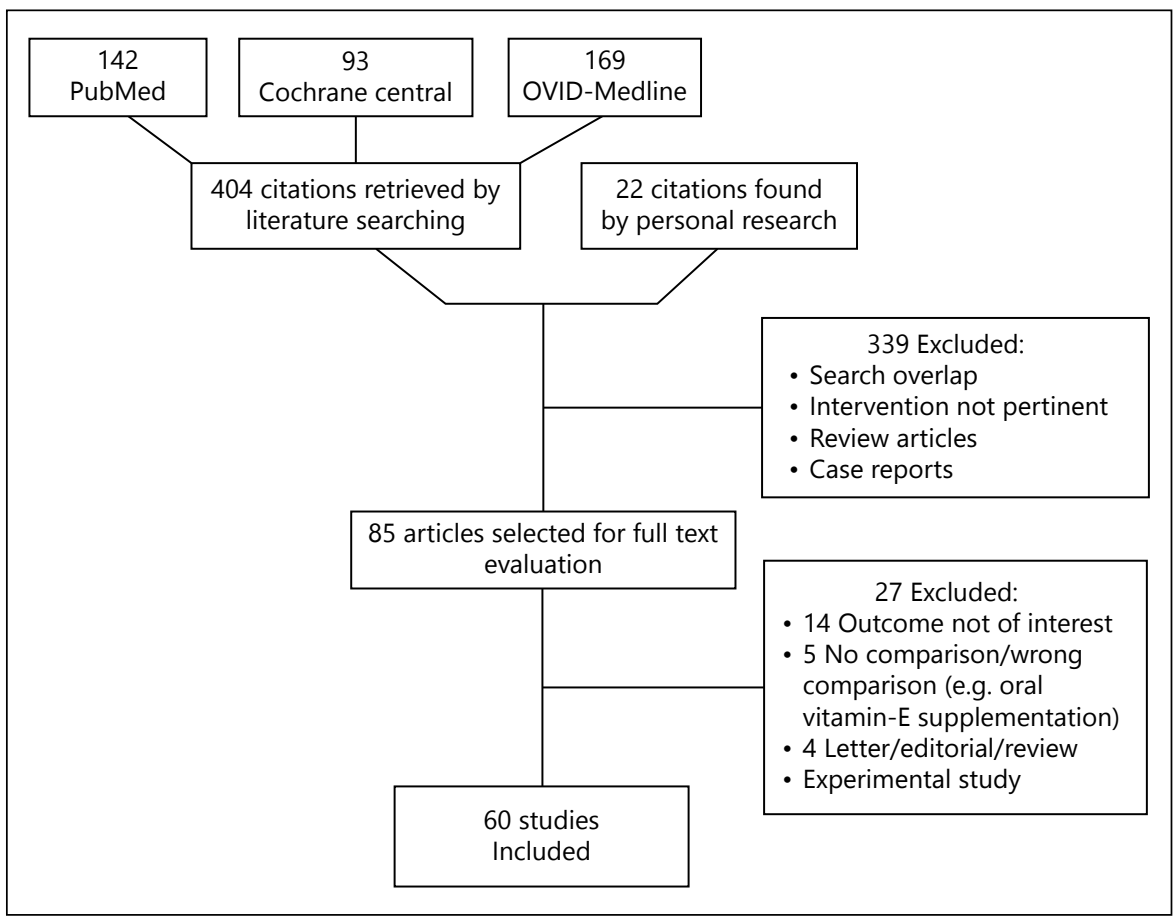

Among the 60 selected studies, 23 were randomized controlled trials of which 10 had a cross-over [14-23] and 13 had a parallel design [10, 24-35] and 37 nonrandomized studies of which 32 had a cross-over [11,36-66] and 5 a parallel design [67-71]. Fifty-two were single-center $[14-20,23-30,32,33,35-59,61-64,66-71]$ and $8[10,11$, $21,22,31,34,60,65]$ were multicenter studies.

The final population analyzed included 2,118 patients, but the range was highly variable across studies, spanning from $7[59,61]$ to 305 [34].

The mean age of participants was 58 spanning from 15 years [61] to 72 [23]. The gender of participants was specified in 50 studies and the percentage of male spanned from $31 \%$ [56] to $86 \%$ [61]. The reported prevalence of diabetes ranged from $0 \%[10,18,31,39,44,46,53,54,63,69]$ to $100 \%$ [22], while hypertension spanned from 9\% [35] to 82\% [70] of the study population. The study duration was not specified in 4 studies [10,39,40,46], while for the other studies, the duration varied from 1 week [26] to 24 months [24, 53]. Dialysis vintage was specified in $73 \%$ of the studies, spanning from 12 months [33] to 158 months [56].

\section{Outcome Data}

Outcome data are summarized in table 1.

The effects of ViE-m on anemia were analyzed in 27 studies $[10,11,16,17,19,21-23,27,32,34,35,42,45,46$, $50,52-55,60-65,69]$.

Vitamin-E vs. Conventional Dialysis: A Meta-Analysis
Changes in hemoglobin $(\mathrm{Hb})$ and $\mathrm{RBCs}$ count were available in $25[10,11,16,17,19,21-23,32,34,35,42,45$, $50,52-55,60-65,69]$ and 6 studies [42, 51, 53-55, 63], respectively. Variations in the percentage of hematocrit (Hct) were provided in 6 studies [42, 46, 51, 53-55]. Five studies $[32,42,51,55,69]$ reported information on total serum iron, $11[17,23,27,32,34,42,51,54,55,64,69]$ on ferritin levels and $6[23,32,34,42,54,55]$ on transferrin saturation (TSAT). Erythropoietin (EPO) dosage was specified in 16 studies $[11,16,19,21,23,27,45,50,51,54$, $55,60,61,64,65,69]$.

Data on EPO resistance index (ERI) was available in 7 studies [11, 21, 23, 32, 34, 35, 62].

Forty-five studies $[10,11,14,16-20,22-26,28-33,36-$ $42,44,46-51,54-58,62-65,67,70,71]$ looked at the effect of ViE-m on markers of oxidative stress. Changes in plasma and RBC glutathione peroxidase (GSH-PX) activities were reported in $3[11,26,44]$ and 5 studies $[36,44,49$, $55,58]$, respectively. Data on RBC superoxide dismutase (SOD) activity were available in 5 studies $[11,26,49,55$, 58]. Plasma malondialdehyde (MDA) was measured in 13 studies $[11,14,18,25,36,40,41,49,50,54,58,63,71]$, while 4 studies [36, 42, 63, 71] provided data on RBC MDA. Twenty one studies $[10,11,17,24,28,30-32,37$, $38,44,46-49,51,54,57,58,63,71]$ examined the effect of ViE-m or conventional membranes on blood vitamin $\mathrm{E}$ levels, while 5 studies $[11,37,42,58,71]$ focused on RBC 
Table 1. Summary of the main effects of ViE-m vs. conventional membranes on parameters pertaining anemia, oxidative stress, inflammation and various clinical endpoints in HD patients

\begin{tabular}{|c|c|c|c|}
\hline Outcome & Parameter & General findings & Sensitivity analysis \\
\hline \multirow[t]{6}{*}{ Anemia } & $\mathrm{Hb}$ & No change & No change \\
\hline & Hct & No change & No change \\
\hline & Total serum iron & No change & No change \\
\hline & TSAT & $\mathrm{MD}-3.59 \%(95 \% \mathrm{CI}-5.44 \text { to }-1.73)^{*}$ & - \\
\hline & EPO dosage & No change & - \\
\hline & ERI & SMD -0.37 ( $95 \%$ CI -0.70 to -0.03$)$ & - \\
\hline \multirow[t]{7}{*}{ Oxidative stress } & Plasma and RBC GSH-PX activity & No change & No change \\
\hline & Blood and $\mathrm{RBC}$ vitamin $\mathrm{E}$ & $\begin{array}{l}\text { Blood vitamin-E: SMD } 0.79(95 \% \text { CI } 0.50 \text { to } 1.08)^{*} \\
\text { RBC vitamin-E: SMD } 0.89(95 \% \text { CI } 0.28 \text { to } 1.50)^{*}\end{array}$ & No change \\
\hline & Blood vitamin $\mathrm{C}$ & No change & - \\
\hline & Plasma Ox-LDL & No change & SMD $-1.26(95 \% \text { CI }-1.79 \text { to }-0.74)^{*}$ \\
\hline & Plasma NOx & No change & - \\
\hline & TBARS & SMD $-1.18(95 \% \text { CI }-1.98 \text { to }-0.38)^{*}$ & No change \\
\hline & TAS and AOC & No change & SMD 0.51 (95\% CI 0.18 to 0.84$)^{*}$ \\
\hline \multirow[t]{2}{*}{ Inflammation } & CRP & No change & - \\
\hline & IL-6 & $\mathrm{MD}-2.25 \mathrm{pg} / \mathrm{ml}(95 \% \mathrm{CI}-3.21 \text { to }-1.30)^{*}$ & - \\
\hline \multirow[t]{7}{*}{ Other } & Total, HDL and LDL cholesterol & No change & - \\
\hline & Triglycerides & No change & - \\
\hline & Pre- and post-dialysis SBP and DBP & No change & - \\
\hline & $\mathrm{Kt} / \mathrm{V}$ & No change & No change \\
\hline & Serum albumin & No change & No change \\
\hline & Uric acid & No change & - \\
\hline & WBC counts & No change & - \\
\hline
\end{tabular}

* Significant results.

vitamin E levels. Six studies [11, 16, 26, 30, 49, 63] focused on blood vitamin C levels. Variation in plasma oxidized low density lipoprotein (Ox-LDL), nitric oxide (NOx) and thiobarbituric acid reactive substances (TBARS) were given in $11[18,19,24,25,46,48,51,56,65,67,70]$, $4[16,22,57,62]$ and 8 studies $[20,26,29,33,39,44,47$, $70]$, respectively. Thirteen studies $[10,20,23,26,30,33$,
$44,48,49,51,58,64,70]$ reported information on total anti-oxidant status (TAS) and anti-oxidant capacity (AOC).

Fifteen studies $[11,15,19,21,23,30,32,34,35,61,64$, $65,68-70]$ investigated the effect of ViE-m on inflammation. Inflammation was evaluated by changes in C-reactive protein (CRP) and interleukin-6 (IL-6) in 14 [11, 19, 
$21,23,30,32,34,35,61,64,65,68-70]$ and 9 studies [ 11 , $15,21,23,32,61,64,68,70]$ respectively.

Finally, 29 studies [11, 15-20, 22, 23, 28, 31, 32, 43, $48-52,54,58,59,62,64,66-71]$ provided information on other patient- or dialysis-related outcomes. Changes in total cholesterol were analyzed in 16 studies $[11,17,18$, $28,31,43,48,50,51,58,64,66-68,70,71]$, high density lipoprotein (HDL) cholesterol in 12 studies [17, 18, 28, $43,48,58,64,66-68,70,71]$, LDL cholesterol in 11 studies $[18,28,43,48,58,64,66-68,70,71]$ and triglycerides in 14 studies $[11,17,18,28,31,43,48,51,58,64,66-68$, 71]. Variations in blood pressure before or after dialysis were available in $5[16,22,32,52,62]$ and 4 studies [22, $32,52,62]$ respectively. Ten studies $[15,16,18,19,23,50$, $51,54,64,69]$ reported information on dialysis adequacy $(\mathrm{Kt} / \mathrm{V})$. Changes in albumin, uric acid and WBC count were available in $10[11,16,17,22,32,50,51,64$, $69,70], 4[49,51,58,64]$ and 5 studies $[18,20,59,64,70]$ respectively.

\section{Effects of ViE-m on Anemia}

Hemoglobin

In data pooled from 17 studies (791 patients) $[16,19$, $21,22,34,42,45,50-55,60,61,63,69]$, ViE-m did not produce significant changes on Hb levels when compared with conventional membrane (MD $0.14 \mathrm{~g} / \mathrm{dl}$; 95\% CI -0.14 to 0.42 ; fig. $2 \mathrm{a}$ ) with high evidence for heterogeneity in the analysis $\left(\mathrm{chi}^{2}=168.76, \mathrm{p}<0.00001 ; \mathrm{I}^{2}=88 \%\right)$. Overall heterogeneity was almost halved $\left(\mathrm{I}^{2}=41 \%\right)$ by excluding studies with longer follow-up period $(>6$ months) $[19,21,34,42,51,53,54,60,69]$.

This absence of effect was in line with isolated findings from 7 studies $[11,17,23,35,62,64,65]$ in which outcome data were not suitable to be included in meta-analysis.

Conversely, in one study [32], a significant increase in $\mathrm{Hb}$ levels was noticed in patients treated either with ViE-m or with conventional membranes.

\section{RBCs Count}

A meta-analysis of 6 non-RCTs [42, 51, 53-55, 63] (107 patients) did not reveal variations in RBC count (MD $101.13 \mathrm{~mm}^{3}$; $95 \% \mathrm{CI}-46.46$ to 248.72 ; fig. $2 \mathrm{~b}$ ) with high heterogeneity $\left(\mathrm{chi}^{2}=18.18, \mathrm{p}=0.003 ; \mathrm{I}^{2}=72 \%\right)$. The study with the highest sample size [51] entirely contributed to the overall heterogeneity of this analysis.

\section{Hematocrit}

ViE-m did not influence Hct levels (6 cross-over nonRCT, 99 patients; MD 1.07\%; 95\% CI -1.17 to 3.30; fig. 3a) $[42,46,51,53-55]$. There was significant heterogeneity in this analysis $\left(\mathrm{chi}^{2}=77.48, \mathrm{p}<0.00001 ; \mathrm{I}^{2}=94 \%\right)$ that was significantly decreased $\left(\mathrm{I}^{2}=32 \%\right)$ after excluding 2 studies conducted on patients with very old dialysis vintage $[51,53]$.

\section{Iron Balance}

In a meta-analysis of 4 non-RCTs [42, 51, 55, 69] (75 patients) ViE-m had no effects on total serum iron (MD $-5.48 \mathrm{mg} / \mathrm{dl} ; 95 \% \mathrm{CI}-15.46$ to 4.51 ; fig. $3 \mathrm{~b}$ ). A sensitivity analysis revealed that the high heterogeneity $\left(\right.$ chi $\left.^{2}=8.45, \mathrm{p}=0.04 ; \mathrm{I}^{2}=64 \%\right)$ can be fully explained by one cross-over study [42] with the longer follow-up period.

Similarly, no effects were reported in one parallel RCT [32] that compared ViE-m with polysulfone dialyzers.

A meta-analysis of 8 studies [23, 27, 42, 51, 54, 55, 64, 69] (139 patients) showed no changes in ferritin levels (MD $5.71 \mathrm{ng} / \mathrm{ml} ; 95 \% \mathrm{CI}-36.49$ to 47.91 ; fig. 3c). This analysis was affected by moderate heterogeneity $\left(\mathrm{chi}^{2}=\right.$ $15.96, \mathrm{p}=0.03 ; \mathrm{I}^{2}=56 \%$ ).

No changes in ferritin were observed in 2 single RCTs $[32,34]$. In contrast, in one cross-over RCT [17], a significant decrease in ferritin levels was observed after switching from conventional to ViE-m.

TSAT was significantly reduced by ViE- $\mathrm{m}$ in a metaanalysis of 4 studies [23, 42, 54, 55] (42 patients) $\left(\mathrm{MD}-3.63 \%\right.$; $95 \% \mathrm{CI}-5.52$ to $-1.74 ; \mathrm{chi}^{2}=2.47, \mathrm{p}=$ 0.48 ; $\mathrm{I}^{2}=0 \%$; fig. $3 \mathrm{~d}$ ), but this finding was not consistent with results from 2 RCTs [32, 34], showing no changes in TSAT.

\section{EPO Dosage}

ViE-m had no effects over conventional membrane on the needed dose of EPO (14 studies, 447 patients; SMD -0.11 ; $95 \%$ CI -0.31 to 0.10 ; fig. 4 a) $[16,19,21,23,27,45$, $50,51,54,55,60,61,64,69]$ with low heterogeneity $\left(\mathrm{chi}^{2}=\right.$ $\left.22.15, \mathrm{p}=0.10 ; \mathrm{I}^{2}=32 \%\right)$.

EPO dosage was unaffected in patients treated with ViE-m in a single cross-over non-RCT [65] and reduced in another [11].

\section{EPO Resistance Index}

ERI improved after ViE-m treatment (3 studies, 286 patients; SMD -0.37 ; $95 \%$ CI -0.70 to -0.03 ; fig. $4 \mathrm{~b}$ ) [21, $32,34]$, with mild heterogeneity in the analysis $\left(\right.$ chi $^{2}=$ $\left.6.15, \mathrm{p}=0.10 ; \mathrm{I}^{2}=51 \%\right)$. The same finding was observed in one RCT [23]. On the contrary, in another RCT [35] and in 2 cross-over studies [11,62], ViE-m had no effects on ERI. 


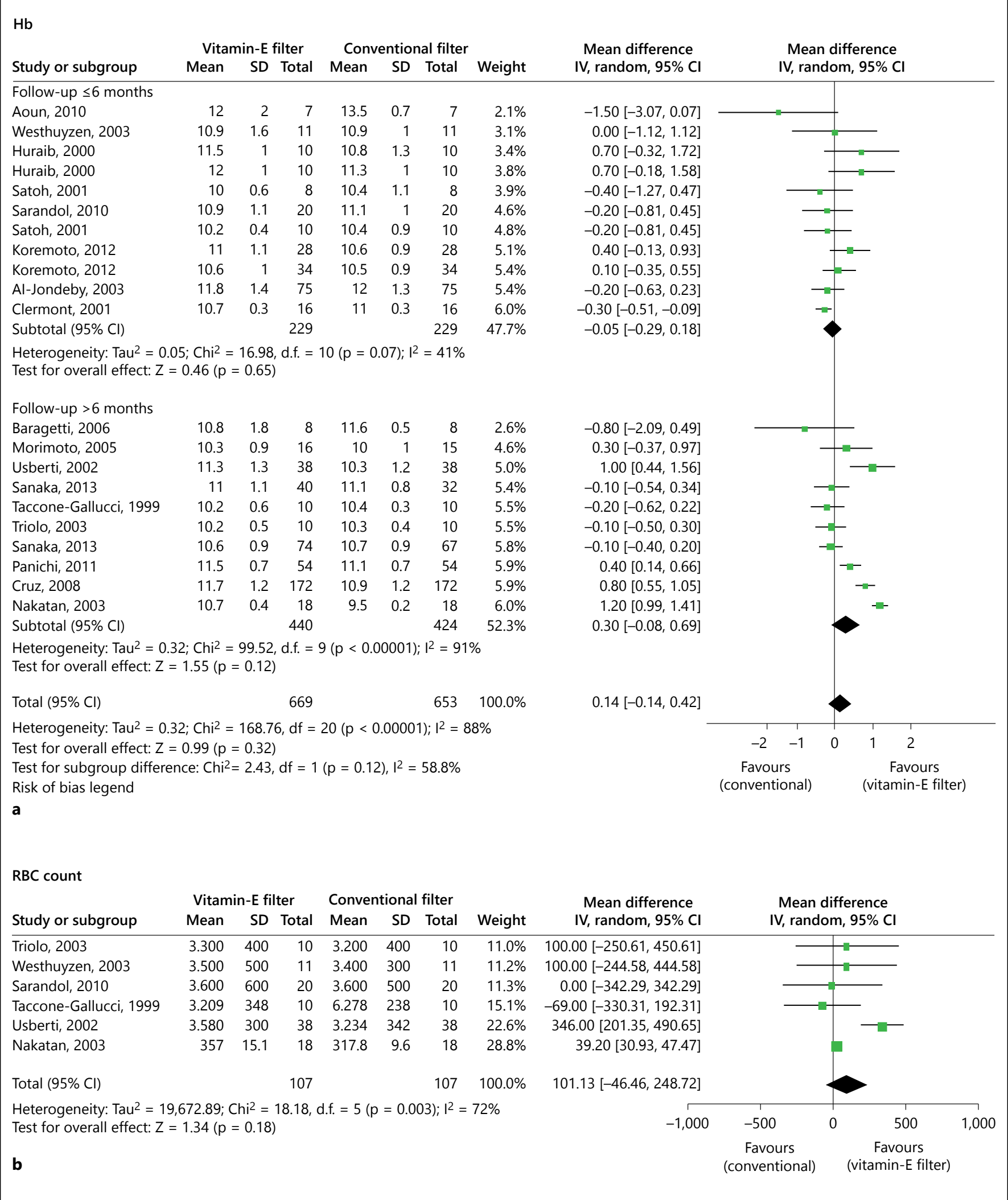

Fig. 2. Effects of ViE-m vs. conventional membrane on Hb levels (a) and RBCs count (b). 
Hct

\begin{tabular}{|c|c|c|c|c|c|c|c|c|c|c|}
\hline \multirow[b]{2}{*}{ Study or subgroup } & \multicolumn{3}{|c|}{ Vitamin-E filter } & \multicolumn{4}{|c|}{ Conventional filter } & \multirow{2}{*}{$\begin{array}{l}\text { Mean difference } \\
\text { IV, random, } 95 \% \mathrm{Cl}\end{array}$} & \multirow{2}{*}{\multicolumn{2}{|c|}{$\begin{array}{c}\text { Mean difference } \\
\text { IV, random, } 95 \% \mathrm{CI}\end{array}$}} \\
\hline & Mean & SD & Total & Mean & SD & Total & Weight & & & \\
\hline Westhuyzen, 2003 & 34 & 5 & 11 & 34 & 3 & 11 & $12.9 \%$ & $0.00[-3.45,3.45]$ & & \\
\hline Miyazaki, 2000 & 34 & 2 & 12 & 36 & 2 & 12 & $17.0 \%$ & $-2.00[-3.60,-0.40]$ & ——- & \\
\hline Triolo, 2003 & 32.9 & 1.7 & 10 & 32.7 & 1.6 & 10 & $17.2 \%$ & $0.20[-1.25,1.65]$ & & \\
\hline Taccone-Gallucci, 1999 & 31.4 & 1.8 & 10 & 31.7 & 1.4 & 10 & $17.3 \%$ & $-0.30[-1.71,1.11]$ & & \\
\hline Usberti, 2002 & 34 & 3 & 38 & 30 & 3.2 & 38 & $17.3 \%$ & $4.00[2.61,5.39]$ & & - \\
\hline Nakatan, 2003 & 34.5 & 1.4 & 18 & 30.5 & 0.7 & 18 & $18.2 \%$ & $4.00[3.28,4.72]$ & & -- \\
\hline Total $(95 \% \mathrm{Cl})$ & & & 99 & & & 99 & $100.0 \%$ & $1.07[-1.17,3.30]$ & & \\
\hline \multicolumn{9}{|c|}{$\begin{array}{l}\text { Heterogeneity: } \text { Tau }^{2}=6.98 ; C \mathrm{Chi}^{2}=77.48, \text { d.f. }=5(\mathrm{p}<0.00001) ; \mathrm{I}^{2}=94 \% \\
\text { Test for overall effect: } Z=0.94(p=0.35)\end{array}$} & $\begin{array}{cc}1 & 1 \\
-4 & -2\end{array}$ & 4 \\
\hline
\end{tabular}

a

Favours (conventional) Favours (vit-E filter)

Total serum iron

\begin{tabular}{|c|c|c|c|c|c|c|c|c|c|c|c|c|c|}
\hline \multirow[b]{2}{*}{ Study or subgroup } & \multicolumn{3}{|c|}{ Vitamin-E filter } & \multicolumn{4}{|c|}{ Conventional filter } & \multirow{2}{*}{$\begin{array}{l}\text { Mean difference } \\
\mathrm{IV} \text {, random, } 95 \% \mathrm{CI}\end{array}$} & \multirow{2}{*}{\multicolumn{5}{|c|}{$\begin{array}{l}\text { Mean difference } \\
\mathrm{IV} \text {, random, } 95 \% \mathrm{Cl}\end{array}$}} \\
\hline & Mean & SD & Total & Mean & SD & Total & Weight & & & & & & \\
\hline Baragetti, 2006 & 61.3 & 8.3 & 8 & 62.2 & 6 & 8 & $36.2 \%$ & $-0.90[-8.00,6.20]$ & & & & & \\
\hline Taccone-Gallucci, 1999 & 73.4 & 28.6 & 10 & 107 & 23.7 & 10 & $13.2 \%$ & $-33.60[-56.62,-10.58]$ & & & & & \\
\hline Usberti, 2002 & 67.7 & 15 & 38 & 66.2 & 19 & 38 & $35.1 \%$ & $1.50[-6.20,9.20]$ & & & - & & \\
\hline Westhuyzen, 2003 & 60 & 25 & 11 & 68 & 24 & 11 & $15.5 \%$ & $-8.00[-28.48,12.48]$ & & & & & \\
\hline Total $(95 \% \mathrm{Cl})$ & & & 67 & & & 67 & $100.0 \%$ & $-5.48[-15.46,4.51]$ & & & & & \\
\hline \multicolumn{9}{|c|}{$\begin{array}{l}\text { Heterogeneity: } \text { Tau }^{2}=58.58 ; C^{2}{ }^{2}=8.45, \text { d.f. }=3(p=0.04) ; 1^{2}=64 \% \\
\text { Test for overall effect: } Z=1.07(p=0.28)\end{array}$} & -50 & $\begin{array}{c}1 \\
-25\end{array}$ & 0 & 25 & 50 \\
\hline b & & & & & & & & & $\begin{array}{r}\text { Fa } \\
\text { (vitam }\end{array}$ & $\begin{array}{l}\text { ours } \\
- \text {-E filter) }\end{array}$ & & $\begin{array}{r}\text { Favo } \\
\text { (conven }\end{array}$ & al) \\
\hline
\end{tabular}

Ferritin

\begin{tabular}{|c|c|c|c|c|c|c|c|c|c|c|c|c|c|}
\hline \multirow{3}{*}{$\frac{\text { Study or subgroup }}{\text { Takouli, } 2010}$} & \multicolumn{3}{|c|}{ Vitamin-E filter } & \multicolumn{3}{|c|}{ Conventional filter } & \multirow[b]{2}{*}{ Weight } & \multirow{2}{*}{$\begin{array}{l}\text { Mean difference } \\
\mathrm{IV} \text {, random, } 95 \% \mathrm{Cl}\end{array}$} & \multirow{2}{*}{\multicolumn{4}{|c|}{$\begin{array}{l}\text { Mean difference } \\
\text { IV, random, } 95 \% \mathrm{Cl}\end{array}$}} & \\
\hline & Mean & SD & Total & Mean & SD & Total & & & & & & & \\
\hline & 672.8 & 410.8 & 9 & 315.5 & 132.5 & 9 & $2.1 \%$ & $357.30[75.30,639.30]$ & & & & 5 & \\
\hline Mandolfo, 2012 & 509 & 241 & 8 & 253 & 198 & 8 & $3.4 \%$ & $256.00[39.86,472.14]$ & & & & & \\
\hline Taccone-Gallucci, 1999 & 361 & 242 & 10 & 368 & 176 & 10 & $4.5 \%$ & $-7.00[-192.46,178.46]$ & & & & & \\
\hline Triolo, 2003 & 309 & 174 & 10 & 342 & 144 & 10 & $7.1 \%$ & $-33.00[-172.99,106.99]$ & & & - & & \\
\hline Baragetti, 2006 & 266.7 & 76.4 & 8 & 257.6 & 102.9 & 8 & $13.2 \%$ & $9.10[-79.71,97.91]$ & & & $1-$ & & \\
\hline Westhuyzen, 2003 & 436 & 78.5 & 11 & 514 & 109.7 & 11 & $14.9 \%$ & $-78.00[-157.72,1.72]$ & & - & & & \\
\hline Usberti, 2002a & 220 & 80 & 38 & 208 & 71 & 38 & $26.5 \%$ & $12.00[-22.01,46.01]$ & & & + & & \\
\hline Kobayashi, 2003 & 130 & 40 & 17 & 132 & 41 & 17 & $28.3 \%$ & $-2.00[-29.23,25.23]$ & & & 1 & & \\
\hline Total $(95 \% \mathrm{Cl})$ & & & 111 & & & 111 & $100.0 \%$ & $5.71[-36.49,47.91]$ & & & 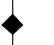 & & \\
\hline \multicolumn{8}{|c|}{$\begin{array}{l}\text { Heterogeneity: } \text { Tau }^{2}=1,447.35 ; \mathrm{Chi}^{2}=15.96, \text { d.f. }=7(p=0.03) ; 1^{2}=56 \% \\
\text { Test for overall effect: } Z=0.27(p=0.79)\end{array}$} & $-1,000$ & -500 & & 0 & 500 & 1,000 \\
\hline c & & & & & & & & & $\begin{array}{r}\text { Favour } \\
\text { (conventi }\end{array}$ & & & $\begin{array}{r}\text { Favo } \\
\text { (vitamin- }\end{array}$ & \\
\hline
\end{tabular}

TSAT

\begin{tabular}{|c|c|c|c|c|c|c|c|c|c|c|c|}
\hline \multirow{3}{*}{ Study or subgroup } & \multicolumn{3}{|c|}{ Vitamin-E filter } & \multicolumn{4}{|c|}{ Conventional filter } & \multirow{2}{*}{$\begin{array}{l}\text { Mean difference } \\
\mathrm{IV} \text {, random, } 95 \% \mathrm{CI}\end{array}$} & \multirow{2}{*}{\multicolumn{3}{|c|}{$\begin{array}{l}\text { Mean difference } \\
\mathrm{IV} \text {, random, } 95 \% \mathrm{Cl}\end{array}$}} \\
\hline & Mean & SD & Total & Mean & SD & Total & Weight & & & & \\
\hline & 27.1 & 6.5 & 8 & 34.7 & 7.4 & 8 & $7.6 \%$ & $-7.60[-14.43,-0.77]$ & & & \\
\hline Triolo, 2003 & 31.4 & 5.1 & 10 & 36.3 & 3.2 & 10 & $25.6 \%$ & $-4.90[-8.63,-1.17]$ & -1 & & \\
\hline Taccone-Gallucci, 1999 & 30.1 & 4.1 & 10 & 33.2 & 4.1 & 10 & $27.6 \%$ & $-3.10[-6.69,0.49]$ & - & & \\
\hline Westhuyzen, 2003 & 23.2 & 4.6 & 11 & 25.6 & 2.2 & 11 & $39.2 \%$ & $-2.40[-5.41,0.61]$ & & & \\
\hline Total $(95 \% \mathrm{Cl})$ & & & 39 & & & 39 & $100.0 \%$ & $-3.63[-5.52,-1.74]$ & & & \\
\hline \multicolumn{9}{|c|}{$\begin{array}{l}\text { Heterogeneity: } \text { Tau }^{2}=0.00 ; \mathrm{Chi}^{2}=2.47, \text { d.f. }=3(p=0.48) ; 1^{2}=0 \% \\
\text { Test for overall effect: } Z=3.77(p=0.002)\end{array}$} & $-10 \quad-5$ & 5 & 10 \\
\hline d & & & & & & & & & $\begin{array}{l}\text { Favours } \\
\text { (vitamin-E filter) }\end{array}$ & $\begin{array}{l}\text { Fav } \\
\text { (conve }\end{array}$ & $\begin{array}{l}\text { ivours } \\
\text { (entional) }\end{array}$ \\
\hline
\end{tabular}

Fig. 3. Effects of ViE-m vs. conventional membrane on Hct levels (a), total serum iron (b), ferritin levels (c) and TSAT (d).

Vitamin-E vs. Conventional Dialysis:

A Meta-Analysis
Blood Purif 2017;43:101-122

DOI: $10.1159 / 000453444$ 


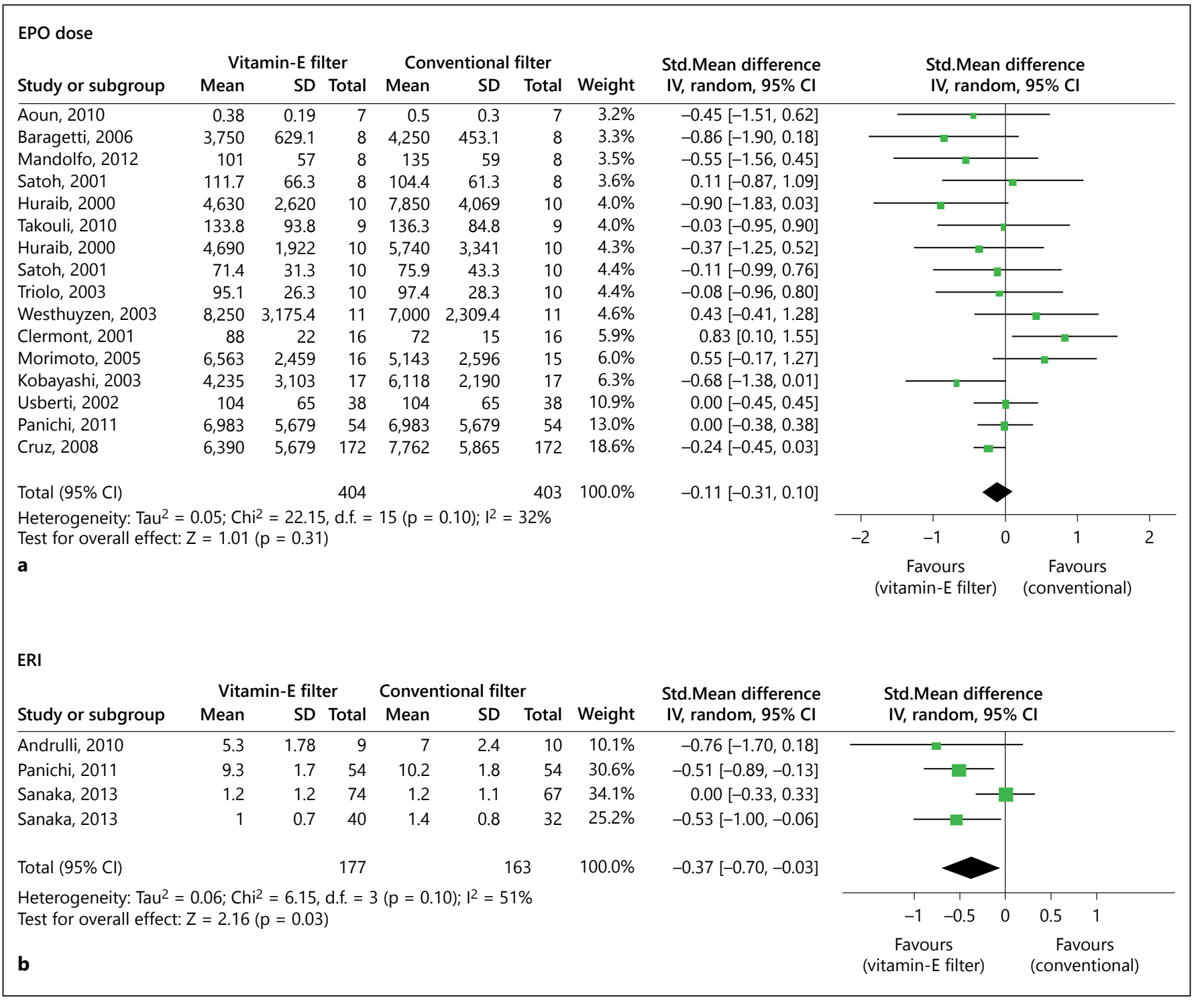

Fig. 4. a, b Effects of ViE-m vs. conventional membrane on EPO dosage.

Effects of ViE-m on Oxidative Stress

Plasma and RBC GSH-PX Activity

ViE-m did not affect plasma GSH-PX activity (3 studies, 66 patients; SMD 0.10; 95\% CI -0.26 to 0.46 ; fig. $5 \mathrm{a}$ ) $[11,26,44]$, with no heterogeneity in the analysis $\left(\mathrm{chi}^{2}=\right.$ $\left.0.39, \mathrm{p}=0.82 ; \mathrm{I}^{2}=0 \%\right)$.

Similarly, in a meta-analysis of 4 cross-over non-RCTs [36, 44, 49, 55], RBC GSH-PX activity remained unchanged (41 patients, MD $6.59 \mathrm{U} / \mathrm{g} \mathrm{Hb}$; 95\% CI -7.26 to 20.45 ; fig. 5b). There was high heterogeneity in this analysis $\left(\mathrm{chi}^{2}=46.80, \mathrm{p}<0.00001 ; \mathrm{I}^{2}=94 \%\right)$ that was slightly reduced $\left(\mathrm{I}^{2}=72 \%\right)$ after excluding data from a cross-over non-RCT [49] with very small sample size.
Another cross-over non-RCT [58] not included in the meta-analysis evidenced no changes in RBC GSH-PX activity after switching to ViE-m.

\section{RBC SOD}

ViE-m did not produce significant changes in RBC SOD activity (3 studies, 31 patients; MD $117.31 \mathrm{IU} / \mathrm{g}$ $\mathrm{Hb} ; 95 \% \mathrm{CI}-35.89$ to $270.51 ; \mathrm{chi}^{2}=7.13, \mathrm{p}=0.03 ; \mathrm{I}^{2}=$ $72 \%$; fig. 5c) $[26,49,55]$. RBC SOD activity remained unchanged in another additional study [58] but was significantly increased after 3 months of ViE-m treatment in a cross-over non-RCT [11]. 
Plasma GSH-PX activity

Vitamin-E filter Conventional filter

Study or subgroup

Eiselt, 2001

Bonnefont-Rousselot, 2000

Bargnoux, 2013

Total $(95 \% \mathrm{Cl})$ Mean

$\begin{array}{ll}42.55 & 9.71\end{array}$

tal Mea

SD Total Weight

$49.25 \quad 62.07$

$\begin{array}{rrr}6 & 40.97 & 8.17 \\ 12 & 230.25 & 53.98\end{array}$

$\begin{array}{lll}42 & 206.75 & 68.43\end{array}$

$6 \quad 10.0 \%$

$12 \quad 19.8 \%$

$42 \quad 70.2 \%$

60

$60 \quad 100.0 \%$

Heterogeneity: $\operatorname{Tau}^{2}=0.00 ; \mathrm{Chi}^{2}=0.39$, d.f. $=2(p=0.82) ; 1^{2}=0 \%$

Test for overall effect: $Z=0.54(p=0.59)$

a

\section{RBC GSH-PX activity}

\begin{tabular}{lrrrrrrr} 
& \multicolumn{3}{c}{ Vitamin-E filter } & \multicolumn{3}{c}{ Conventional filter } \\
Study or subgroup & Mean & SD & Total & Mean & SD & Total & Weight \\
\hline Westhuyzen, 2003 & 56.7 & 17.2 & 11 & 46.8 & 14.8 & 11 & $22.1 \%$ \\
Bonnefont-Rousselot, 2000 & 45.7 & 10.7 & 12 & 44.2 & 11.8 & 12 & $24.9 \%$ \\
Mydlik, 2001 & 69.9 & 9.8 & 8 & 48.1 & 7 & 8 & $25.3 \%$ \\
Buoncristiani, 1997 & 34.6 & 1.3 & 10 & 39.9 & 0.4 & 10 & $27.8 \%$ \\
& & & & & & & \\
Total (95\% CI) & & & 41 & & & 41 & $100.0 \%$
\end{tabular}

Total $(95 \% \mathrm{Cl})$

41

$41 \quad 100.0 \%$

Heterogeneity: Tau $^{2}=179.65 ; \mathrm{Chi}^{2}=46.80$, d.f. $=3(p<0.00001) ; \mathrm{I}^{2}=94 \%$

Test for overall effect: $Z=0.93(p=0.35)$

b

RBC SOD

Study or subgroup

Vitamin-E filter Conventional filter

Eiselt, 2001

Mydlik, 2001

Westhuyzen, 2003

Total $(95 \% \mathrm{Cl})$

Heterogeneity: Tau $^{2}=12,803.63 ; \mathrm{Chi}^{2}=7.13, \mathrm{df}=2(\mathrm{p}=0.03) ; \mathrm{I}^{2}=72 \%$

Test for overall effect: $Z=1.50(p=0.13)$

c

Mean SD Total Mean SD Total Weight IV, random, $95 \% \mathrm{Cl}$

IV, random, $95 \% \mathrm{CI}$

$9.90[-3.51,23.31]$

$1.50[-7.51,10.51]$

$21.80[13.45,30.15]$

$-5.30[-6.14,-4.46]$

$6.59[-7.26,20.45]$

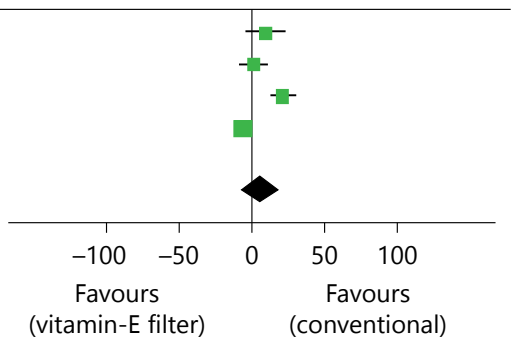

(vitamin-E filter) (conventional)

Std. Mean difference

IV, random, $95 \% \mathrm{Cl}$

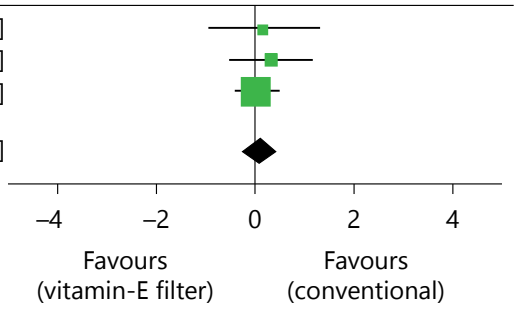

IV, random, $95 \% \mathrm{CI}$

$\begin{array}{llllllll}957.2 & 230.6 & 6 & 930.2 & 134.2 & 6 & 24.8 \% & 27.00[-186.49,240.49]\end{array}$

$\begin{array}{llllllll}771 & 151 & 11 & 725 & 102 & 11 & 38.6 \% & 46.00\end{array}\left[\begin{array}{l}-61.68,153.68\end{array}\right.$

25

$25100.0 \% \quad 117.31[-35.89,270.51]$

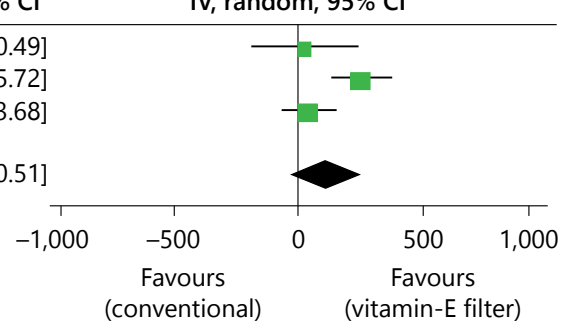

$\begin{array}{lllllllll}912 & 160.5 & 8 & 658.4 & 72.8 & 8 & 36.6 \% & 253.60[131.48,375.72]\end{array}$
Std. Mean difference

IV, random, 95\% Cl

Plasma MDA

Study or subgroup

Satoh, 2001

Shimazu, 1999

Triolo, 2003

Mydlik, 2001

Sommerburg, 1999

Satoh, 2001

Mydlik, 2004

Zhao, 2015

Sarandol, 2010

Racek, 1999

Bargnoux, 2013

Total $(95 \% \mathrm{Cl})$

Vitamin-E filter Conventional filter Mean SD Total Mean SD Total Weight

Std. Mean difference

Std. Mean difference IV, random, $95 \% \mathrm{CI} \quad \mathrm{IV}$, random, $95 \% \mathrm{CI}$

Heterogeneity: Tau $^{2}=0.38 ;$ Chi $^{2}=34.85$, d.f. $=10(p=0.0001) ; 1^{2}=71 \%$

Test for overall effect: $Z=3.73(p=0.0002)$

d

$\begin{array}{rrrrrrr}3.3 & 0.2 & 8 & 4.1 & 0.4 & 8 & 6.1 \% \\ 3.5 & 0.7 & 6 & 4.3 & 0.9 & 5 & 6.5 \% \\ 1.3 & 0.2 & 10 & 1.9 & 0.4 & 10 & 7.7 \% \\ 0.96 & 0.1 & 8 & 1.27 & 0.4 & 8 & 7.8 \% \\ 1.9 & 0.3 & 10 & 2.3 & 0.3 & 10 & 8.3 \% \\ 2.8 & 0.6 & 10 & 3 & 0.3 & 10 & 9.0 \% \\ 2.2 & 0.4 & 14 & 2.8 & 0.4 & 14 & 9.3 \% \\ 4.4 & 1.5 & 20 & 5.7 & 2.1 & 20 & 10.8 \% \\ 1.4 & 0.3 & 20 & 1.5 & 0.4 & 20 & 10.9 \% \\ 3.8 & 0.5 & 24 & 4.2 & 0.6 & 24 & 11.2 \% \\ 3.4 & 1.7 & 42 & 3 & 1.3 & 42 & 12.3 \%\end{array}$

$-2.39[-3.76,-1.02]$

$-0.92[-2.20,0.36]$

$-1.82[-2.90,-0.74]$

$-1.01[-2.06,0.05]$

$-1.28[-2.26,-0.30]$

$-0.40[-1.29,0.48]$

$-1.46[-2.30,-0.61]$

$-0.70[-1.34,-0.06]$

$-0.28[-0.90,0.35]$

$-0.71[-1.30,-0.13]$

$0.26[-0.17,0.69]$

$-0.86[-1.30,-0.41]$

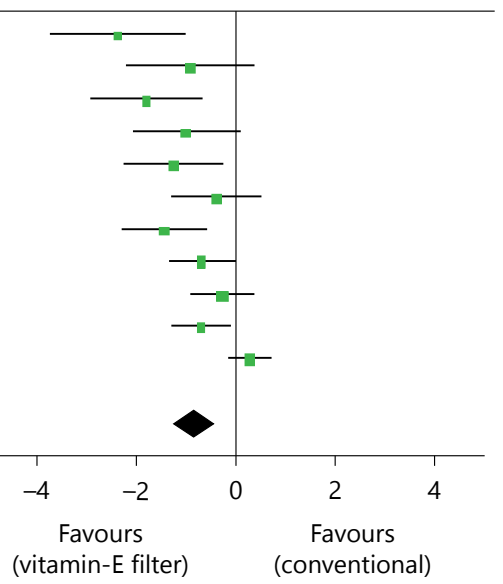

(vitamin-E filter)

(conventional)

Fig. 5. Effects of ViE-m vs. conventional membrane on plasma and RBC GSH-PX activity (a, b), RBC SOD (c) and plasma MDA (d).

Vitamin-E vs. Conventional Dialysis:

A Meta-Analysis
Blood Purif 2017;43:101-122

DOI: $10.1159 / 000453444$ 
Plasma and RBC MDA

Plasma MDA levels were decreased by ViE-m treatment (10 studies, 197 patients; SMD -0.86 ; $95 \%$ CI -1.30 to -0.41 ; fig. 5d) $[11,25,40,41,49,50,54,58,63,71]$. The high heterogeneity observed in this analysis $\left(\mathrm{chi}^{2}=34.85, \mathrm{p}=0.0001\right.$; $\left.\mathrm{I}^{2}=71 \%\right)$ was notably reduced $\left(\mathrm{I}^{2}=41 \%\right)$ after excluding data from the only multicenter cross-over non-RCT [11].

The same effect was observed in 2 RCTs $[14,18]$ and in one non-RCT cross-over [36].

Similarly, ViE-m decreased RBC MDA (3 studies, 70 patients; SMD -2.16 ; $95 \%$ CI -3.88 to $-0.44 ; \mathrm{chi}^{2}=20.63$, $\mathrm{p}<0.0001 ; \mathrm{I}^{2}=90 \%$; fig. 6a) $[42,63,71]$.

Similar results were reported in another cross-over study [36].

Blood and RBC Vitamin E

Blood vitamin $\mathrm{E}$ was increased in patients dialyzed with ViE-m (16 studies, 355 patients; SMD 0.79; 95\% CI 0.50-1.08; fig. 6b) [10, 11, 28, 31, 37, 44, 46-49, 51, 54, 57, $58,63,71]$ with moderate heterogeneity $\left(\mathrm{chi}^{2}=38.94, \mathrm{p}=\right.$ $\left.0.001 ; \mathrm{I}^{2}=59\right)$, which decreased $\left(\mathrm{I}^{2}=36 \%\right)$ when considering studies only with a sample size $\geq 20$ (fig. $6 \mathrm{~b}$ ).

Blood vitamin $\mathrm{E}$ was increased also in 2 additional studies [17, 38]. On the contrary, in 3 studies [24, 30, 32], the use of ViE-m did not influence blood vitamin E levels.

A meta-analysis including 4 studies $[37,42,58,71]$ demonstrated a significant increase in RBC vitamin Elevels after ViE-m treatment (79 patients, SMD 0.89; 95\% CI 0.28-1.50; fig. 6c). One cross-over non-RCT [37] that enrolled mostly elderly patients, fully contributed to explaining the overall moderate heterogeneity $\left(\mathrm{chi}^{2}=7.19, \mathrm{p}=0.07 ; \mathrm{I}^{2}=58 \%\right)$.

A similar increase in RBC vitamin Elevels was observed in another cross-over non-RCT [11].

\section{Blood Vitamin C}

In a meta-analysis including 3 cross-over non-RCTs $[11,49,63]$ and one cross-over RCT [16] (86 patients), the use of ViE-m did not have an impact on blood vitamin C levels (SMD 0.38; $95 \% \mathrm{CI}-0.03$ to $0.79 ; \mathrm{chi}^{2}=4.83$, $\mathrm{p}=$ 0.18 ; $\mathrm{I}^{2}=38 \%$; fig. $7 \mathrm{a}$ ).

Conversely, in 2 other studies $[26,30]$ a significant decrease in blood vitamin $\mathrm{C}$ levels after HD was noticed in each study group.

\section{Plasma Ox-LDL}

ViE-m did not affect Ox-LDL levels (7 studies, 176 patients; SMD $-0.63 ; 95 \% \mathrm{CI}-1.35$ to $0.08 ; \mathrm{chi}^{2}=32.85$, $\mathrm{p}<$ $0.0001 ; I^{2}=82 \%$; fig. 7b) $[19,25,51,56,65,67,70]$. In a sensitivity analysis excluding 3 parallel studies $[25,67$, 70 ], the heterogeneity was more than halved and Ox-LDL levels were significantly reduced $\left(\mathrm{I}^{2}=35 \%\right.$; $\mathrm{SMD}-1.26$; $95 \%$ CI -1.79 to -0.74 ; fig. $7 \mathrm{c}$ ).

Four more studies documented a similar improvement in Ox-LDL $[18,24,46,48]$.

\section{Plasma NOx}

ViE-m did not alter plasma NOx levels (3 studies, 80 patients; MD $-1.08 \mu \mathrm{mol} / \mathrm{l} ; 95 \% \mathrm{CI}-62.40$ to 60.24 , $\mathrm{chi}^{2}=28.74, \mathrm{p}<0.00001 ; \mathrm{I}^{2}=90 \%$; fig. 7d) $[22,57,62]$.

Conversely, NOx levels were increased in one crossover RCT [16].

Thiobarbituric Acid Reactive Substances

In a meta-analysis of 6 studies (117 patients) [20, 26, $39,44,47,70]$, ViE-m reduced significantly TBARS levels (SMD -1.18; 95\% CI -1.98 to -0.38 ; fig. 8a). The high heterogeneity observed $\left(\mathrm{chi}^{2}=22.59, \mathrm{p}=0.0004 ; \mathrm{I}^{2}=78 \%\right)$ was nullified excluding 3 studies [26, 39, 47] mostly focusing on old patients and with short follow-up.

This finding was in accordance with those reported in 2 single studies $[29,33]$.

\section{TAS and AOC}

ViE-m did not influence TAS and AOC ( 9 studies, 183 patients; SMD 0.31 ; $95 \%$ CI -0.17 to 0.80 ; fig. $8 \mathrm{~b}$ ) [10, 20, $26,44,48,49,51,64,70]$. Three studies $[23,33,58]$ reported results that were in line with such findings.

However, TAS and AOC were significantly increased in a sensitivity analysis carried out in order to explain the high heterogeneity $\left(\mathrm{chi}^{2}=27.53, \mathrm{p}=0.001 ; \mathrm{I}^{2}=67 \%\right)$, notincluding a cross-over non-RCT [48] $\left(\mathrm{chi}^{2}=10.50, \mathrm{p}=0.23 ; \mathrm{I}^{2}=24 \%\right.$; SMD 0.51; 95\% CI 0.18-0.84; fig. 8c). TAS and AOC were significantly increased also in another parallel RCT [30].

\section{Effects of ViE-m on Inflammation}

\section{C-Reactive Protein}

CRP levels remained unchanged by ViE-m treatment (8 studies, 254 patients; SMD -0.35 ; $95 \%$ CI -0.79 to 0.09 ; fig. 9a) $[11,19,21,64,65,68-70]$ with high heterogeneity in the analysis $\left(\mathrm{chi}^{2}=27.12, \mathrm{p}=0.0003 ; \mathrm{I}^{2}=74 \%\right)$. Results from 5 additional studies [23, 32, 34, 35, 61] were consistent with this finding. Conversely, one study [30] demonstrated a significant decrease of CRP levels in patients dialyzing with ViE-m.

\section{Interleukin-6}

In a meta-analysis of 5 studies $[11,21,64,68,70], \mathrm{ViE}$ $\mathrm{m}$ induced a significant decrease in IL-6 levels (184 patients; $\mathrm{MD}-2.21 \mathrm{pg} / \mathrm{ml} ; 95 \% \mathrm{CI}-3.01$ to $-1.41 ; \mathrm{chi}^{2}=$ $3.29, \mathrm{p}=0.51 ; \mathrm{I}^{2}=0 \%$; fig. $\left.9 \mathrm{~b}\right)$. 
RBC MDA

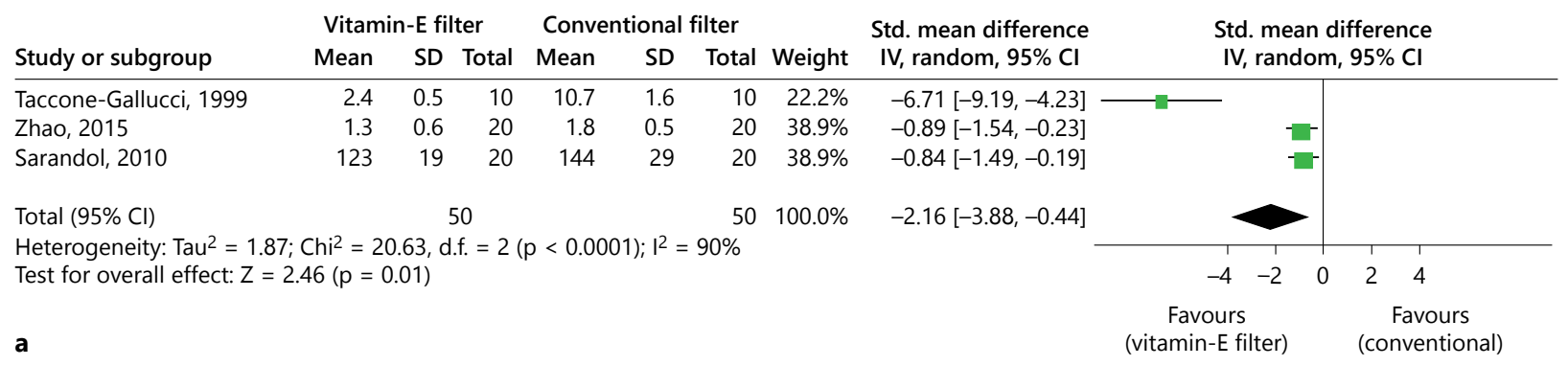

Blood vitamin $\mathrm{E}$

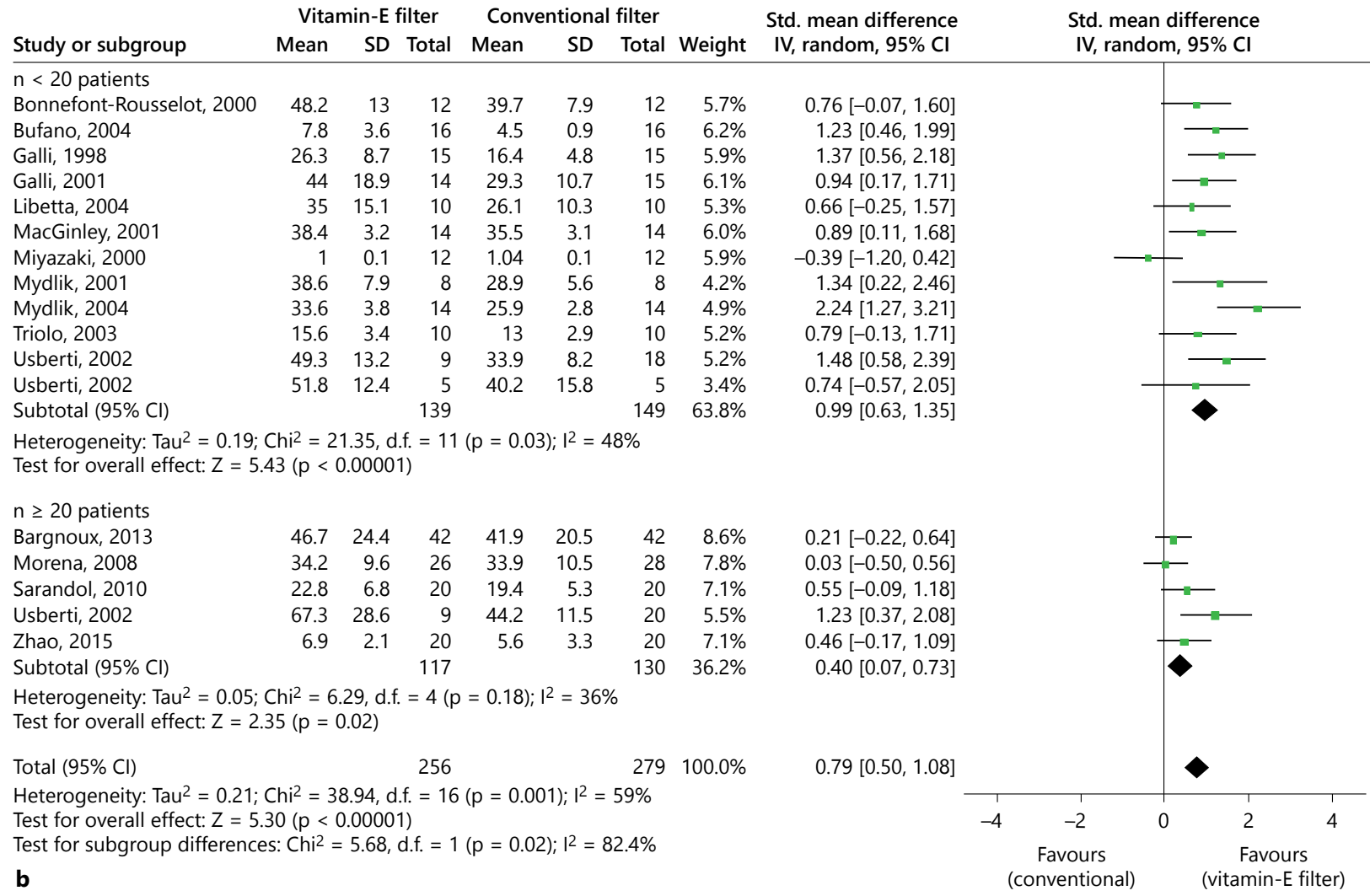

RBC vitamin E

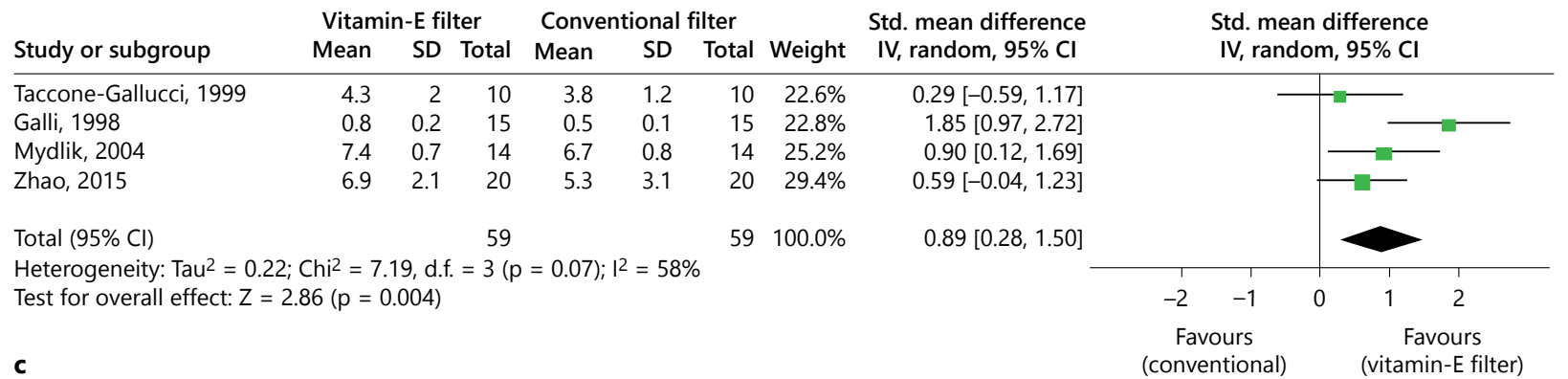

Fig. 6. Effects of ViE-m vs. conventional membrane on RBC MDA (a), blood vitamin $\mathrm{E}$ with sensitivity analysis for exploring causes of heterogeneity (b) and RBC vitamin $\mathrm{E}(\mathbf{c})$.

Vitamin-E vs. Conventional Dialysis:

A Meta-Analysis
Blood Purif 2017;43:101-122

DOI: $10.1159 / 000453444$ 
Blood vitamin C

\begin{tabular}{lrrrrrrr} 
& \multicolumn{3}{c}{ Vitamin-E filter } & \multicolumn{2}{c}{ Conventional filter } \\
Study or subgroup & Mean & SD & Total & Mean & SD & Total & Weight \\
\hline Mydlik, 2001 & 0.5 & 0.2 & 8 & 0.5 & 0.7 & 8 & $14.0 \%$ \\
Clermont, 2001 & 7 & 1.5 & 16 & 5.5 & 1.3 & 16 & $21.0 \%$ \\
Sarandol, 2010 & 0.7 & 0.6 & 20 & 0.5 & 0.3 & 20 & $26.2 \%$ \\
Bargnoux, 2013 & 0.8 & 0.8 & 42 & 0.7 & 0.7 & 42 & $38.7 \%$ \\
Total (95\% Cl) & & & & & & & \\
S & & & 86 & & & 86 & $100.0 \%$
\end{tabular}

Heterogeneity: Tau $^{2}=0.07 ; \mathrm{Chi}^{2}=4.83$, d.f. $=3(p=0.18) ;\left.\right|^{2}=38 \%$

Test for overall effect: $Z=1.79(p=0.07)$

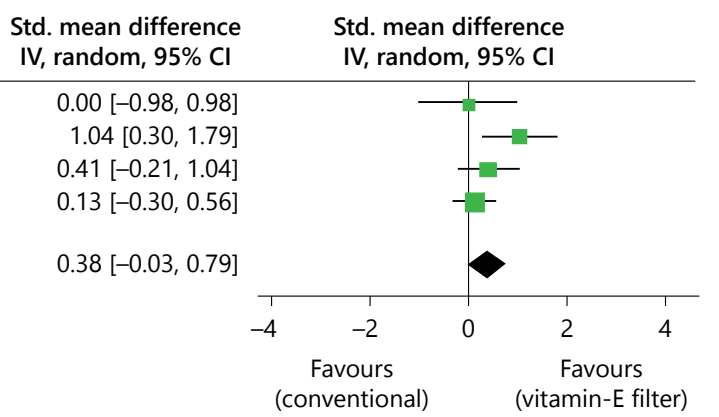

Plasma Ox-LDL

\begin{tabular}{lrrrrrrr} 
& \multicolumn{3}{c}{ Vitamin-E filter } & \multicolumn{2}{c}{ Conventional filter } \\
Study or subgroup & Mean & SD & Total & Mean & SD & Total & Weight \\
\hline Calò, 2011 & 222.1 & 45.2 & 23 & 373.7 & 101.3 & 23 & $15.5 \%$ \\
Hara, 2004 & 4.6 & 1.7 & 13 & 6.6 & 1.7 & 13 & $14.6 \%$ \\
Kirmizis, 2011 & 33 & 8 & 35 & 40 & 20 & 25 & $16.6 \%$ \\
Morimoto, 2005 & 1.3 & 0.5 & 16 & 1.9 & 0.8 & 15 & $15.2 \%$ \\
Shimazu, 1999 & 1.4 & 0.8 & 6 & 1.6 & 0.8 & 5 & $12.1 \%$ \\
Takenaka, 2002 & 2.1 & 0.4 & 14 & 1.7 & 0.3 & 14 & $14.8 \%$ \\
Usberti, 2002 & 169 & 150 & 5 & 361 & 250 & 5 & $11.2 \%$
\end{tabular}

Std. mean difference Std. mean rifference

IV, random, $95 \% \mathrm{Cl} \quad \mathrm{IV}$, random, $95 \% \mathrm{CI}$

$-1.90[-2.61,-1.19]$

$-1.14[-1.98,-0.30]$

$-0.48[-1.01,0.04]$

$-0.88[-1.63,-0.14]$

$-0.23[-1.42,0.96]$

$1.10[0.30,1.90]$

$-0.84[-2.17,0.49]$

$\begin{array}{llll}\text { Total }(95 \% \mathrm{Cl}) & 112 & 100 & 100.0 \%\end{array}$

Heterogeneity: Tau $^{2}=0.73 ; \mathrm{Chi}^{2}=32.85$, d.f. $=6(p<0.0001) ;\left.\right|^{2}=82 \%$

Test for overall effect: $Z=1.73(p=0.08)$

b

$-0.63[-1.35,0.08]$

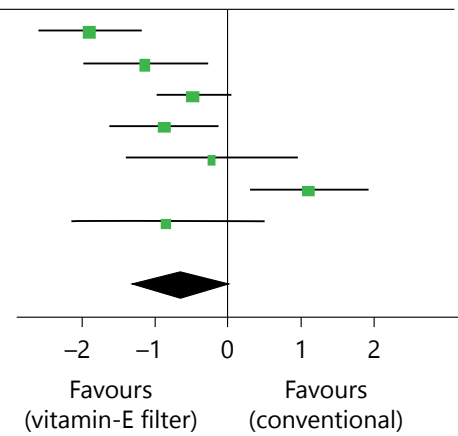

Plasma Ox-LDL

\begin{tabular}{lrrrrrrr} 
& \multicolumn{3}{c}{ Vitamin-E filter } & \multicolumn{2}{c}{ Conventional filter } \\
Study or subgroup & Mean & SD & Total & Mean & SD & Total & Weight \\
\hline Calò, 2011 & 222.1 & 45.2 & 23 & 373.7 & 101.3 & 23 & $31.6 \%$ \\
Hara, 2004 & 4.6 & 1.7 & 13 & 6.6 & 1.7 & 13 & $25.6 \%$ \\
Kirmizis, 2011 & 33 & 8 & 35 & 40 & 20 & 25 & $0.0 \%$ \\
Morimoto, 2005 & 1.3 & 0.5 & 16 & 1.9 & 0.8 & 15 & $29.8 \%$ \\
Shimazu, 1999 & 1.4 & 0.8 & 6 & 1.6 & 0.8 & 5 & $0.0 \%$ \\
Takenaka, 2002 & 2.1 & 0.4 & 14 & 1.7 & 0.3 & 14 & $0.0 \%$ \\
Usberti, 2002 & 169 & 150 & 5 & 361 & 250 & 5 & $13.0 \%$
\end{tabular}

Std. mean difference Std. mean difference IV, random, $95 \% \mathrm{CI}$

-1.90 [-2.61, -1.19]

$-1.14[-1.98,-0.30]$

$-0.48[-1.01,0.04]$

$-0.88[-1.63,-0.14]$

$-0.23[-1.42,0.96]$

$1.10[0.30,1.90]$

$-0.84[-2.17,0.49]$

$-1.26[-1.79,-0.74]$

Total $(95 \% \mathrm{Cl})$

57

$56 \quad 100.0 \%$

Heterogeneity: Tau $^{2}=0.10 ;$ Chi $^{2}=4.58$, d.f. $=3(p=0.21) ;\left.\right|^{2}=35 \%$

Test for overall effect: $Z=4.70(p<0.00001)$

C

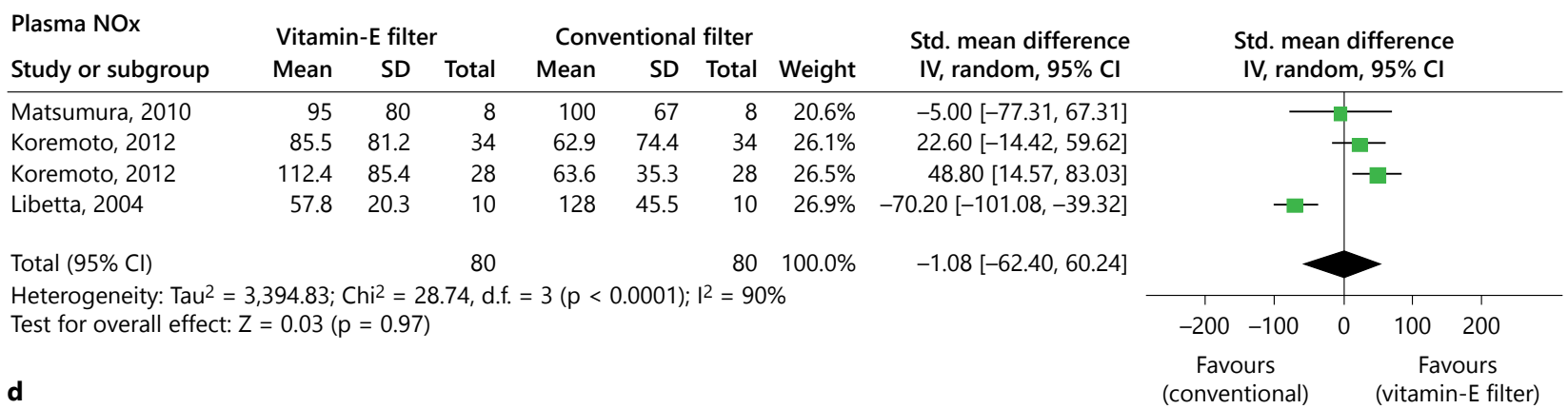

Fig. 7. Effects of ViE-m vs. conventional membrane on blood vitamin $\mathrm{C}$ (a) and plasma Ox-LDL (b) with sensitivity analysis for exploring causes of heterogeneity (c). Effects of ViE-m vs. conventional membrane on plasma NOx (d). 
TBARS

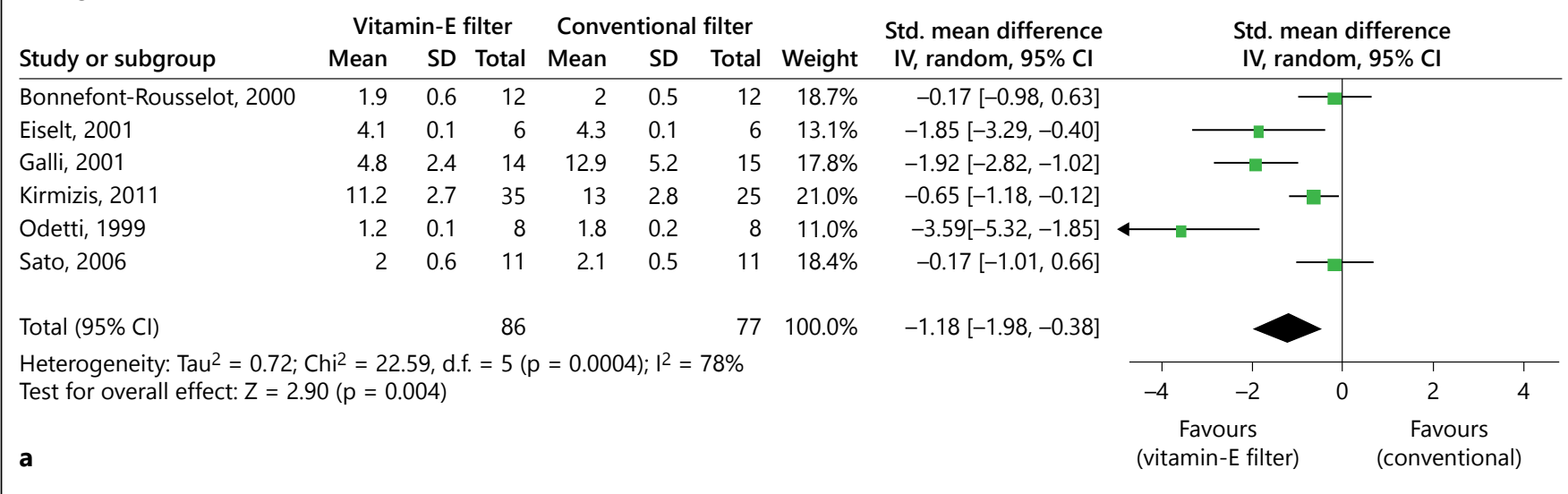

\section{TAS and AOC}

\begin{tabular}{|c|c|c|c|c|c|c|c|}
\hline \multirow[b]{2}{*}{ Study or subgroup } & \multicolumn{3}{|c|}{ Vitamin-E filter } & \multicolumn{3}{|c|}{ Conventional filter } & \multirow[b]{2}{*}{ Weight } \\
\hline & Mean & SD & Total & Mean & SD & Total & \\
\hline Bonnefont-Rousselot, 2000 & 1 & 0.3 & 12 & 1 & 0.2 & 12 & $10.9 \%$ \\
\hline Eiselt, 2001 & 1.4 & 0.03 & 6 & 1.4 & 0.02 & 6 & $8.4 \%$ \\
\hline Kirmizis, 2011 & 1.7 & 0.2 & 35 & 1.6 & 0.1 & 25 & $13.1 \%$ \\
\hline MacGinley, 2001 & 1.5 & 0.05 & 14 & 1.6 & 0.09 & 14 & $10.7 \%$ \\
\hline Mydlik, 2001 & 1.8 & 0.2 & 8 & 1.4 & 0.2 & 8 & $7.7 \%$ \\
\hline Sato, 2006 & 1.2 & 0.1 & 11 & 1.1 & 0.1 & 11 & $10.2 \%$ \\
\hline Takouli, 2010 & 1.5 & 0.3 & 9 & 1.3 & 0.3 & 9 & $9.7 \%$ \\
\hline Usberti, 2002 & 1.4 & 0.2 & 9 & 1.3 & 0.2 & 18 & $10.8 \%$ \\
\hline Usberti, 2002 & 1.4 & 0.2 & 9 & 1.3 & 0.2 & 20 & $10.9 \%$ \\
\hline Usberti, 2002 & 1.3 & 0.2 & 5 & 1.4 & 0.2 & 5 & $7.5 \%$ \\
\hline Total $(95 \% \mathrm{Cl})$ & & & 118 & & & 128 & $100.0 \%$ \\
\hline \multicolumn{8}{|c|}{$\begin{array}{l}\text { Heterogeneity: } \text { Tau }^{2}=0.39 ; \mathrm{Chi}^{2}=27.53, \text { d.f. }=9(p=0.001) ; 1^{2}=67 \% \\
\text { Test for overall effect: } Z=1.26(p=0.21)\end{array}$} \\
\hline
\end{tabular}

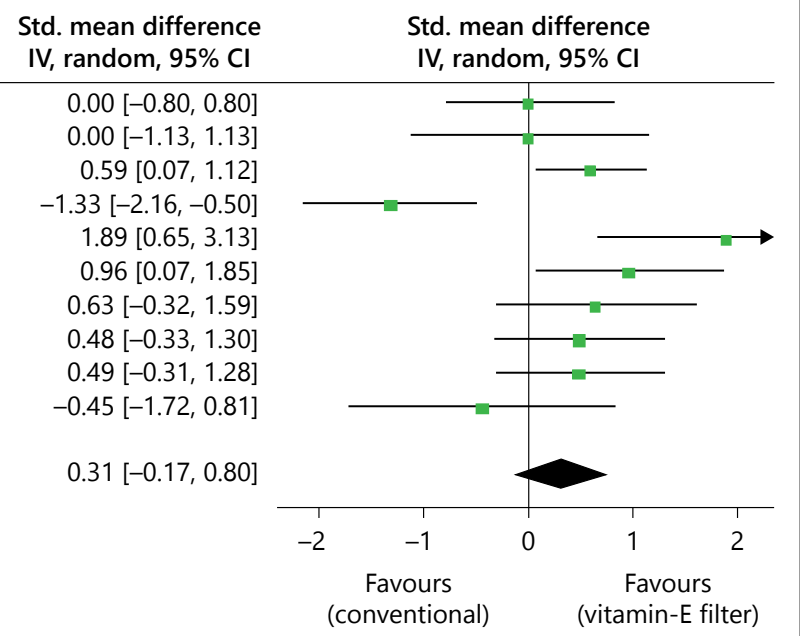

\section{TAS and AOC}

\begin{tabular}{|c|c|c|c|c|c|c|c|}
\hline \multirow[b]{2}{*}{ Study or subgroup } & \multicolumn{3}{|c|}{ Vitamin-E filter } & \multicolumn{3}{|c|}{ Conventional filter } & \multirow[b]{2}{*}{ Weight } \\
\hline & Mean & SD & Total & Mean & SD & Total & \\
\hline Bonnefont-Rousselot, 2000 & 1 & 0.3 & 12 & 1 & 0.2 & 12 & $12.7 \%$ \\
\hline Eiselt, 2001 & 1.4 & 0.03 & 6 & 1.4 & 0.02 & 6 & $7.3 \%$ \\
\hline Kirmizis, 2011 & 1.7 & 0.2 & 35 & 1.6 & 0.1 & 25 & $21.8 \%$ \\
\hline MacGinley, 2001 & 1.5 & 0.05 & 14 & 1.6 & 0.09 & 14 & \\
\hline Mydlik, 2001 & 1.8 & 0.2 & 8 & 1.4 & 0.2 & 8 & $6.3 \%$ \\
\hline Sato, 2006 & 1.2 & 0.1 & 11 & 1.1 & 0.1 & 11 & $10.8 \%$ \\
\hline Takouli, 2010 & 1.5 & 0.3 & 9 & 1.3 & 0.3 & 9 & $9.7 \%$ \\
\hline Usberti, 2002 & 1.4 & 0.2 & 9 & 1.3 & 0.2 & 20 & $12.8 \%$ \\
\hline Usber & 1.4 & 0.2 & 9 & 1.3 & 0.2 & 18 & $12.4 \%$ \\
\hline Usberti, 2002 & 1.3 & 0.2 & 5 & 1.4 & 0.2 & 5 & $6.1 \%$ \\
\hline Total $(95 \% \mathrm{Cl})$ & & & 104 & & & 114 & $100.0 \%$ \\
\hline
\end{tabular}

Std. mean difference Std. mean difference

IV, random, $95 \%$

$0.00[-0.80,0.80]$

$0.00[-1.13,1.13]$

$0.59[0.07,1.12]$

Not estimable

$1.89[0.65,3.13]$

$0.96[0.07,1.85]$

$0.63[-0.32,1.59]$

$0.49[-0.31,1.28]$

$0.48[-0.33,1.30]$

$-0.45[-1.72,0.81]$

$0.51[0.18,0.84]$

Test for overall effect: $Z=3.00(p=0.003)$

C

IV, random, $95 \% \mathrm{Cl}$

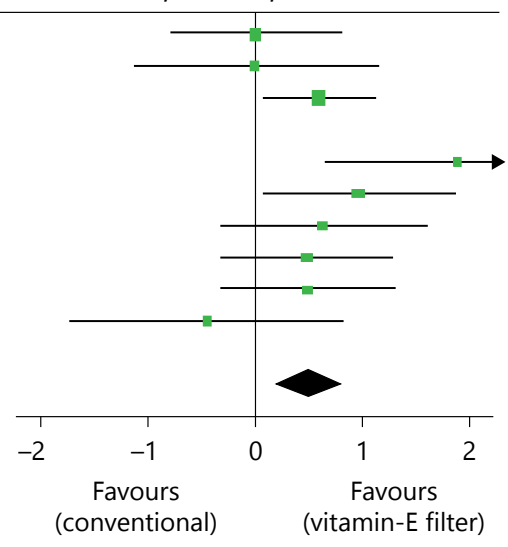

Fig. 8. Effects of ViE-m vs. conventional membrane on TBARS (a) and TAS and AOC (b) with sensitivity analysis for exploring causes of heterogeneity $(\mathbf{c})$. 
CRP

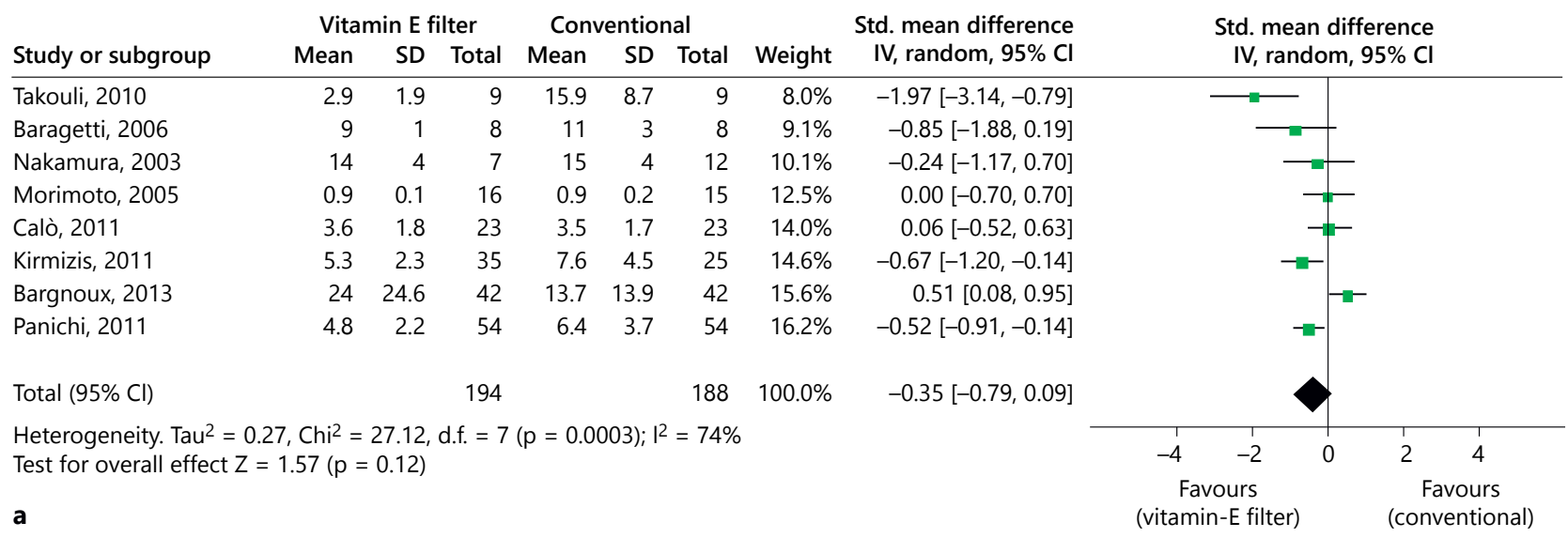

IL-6

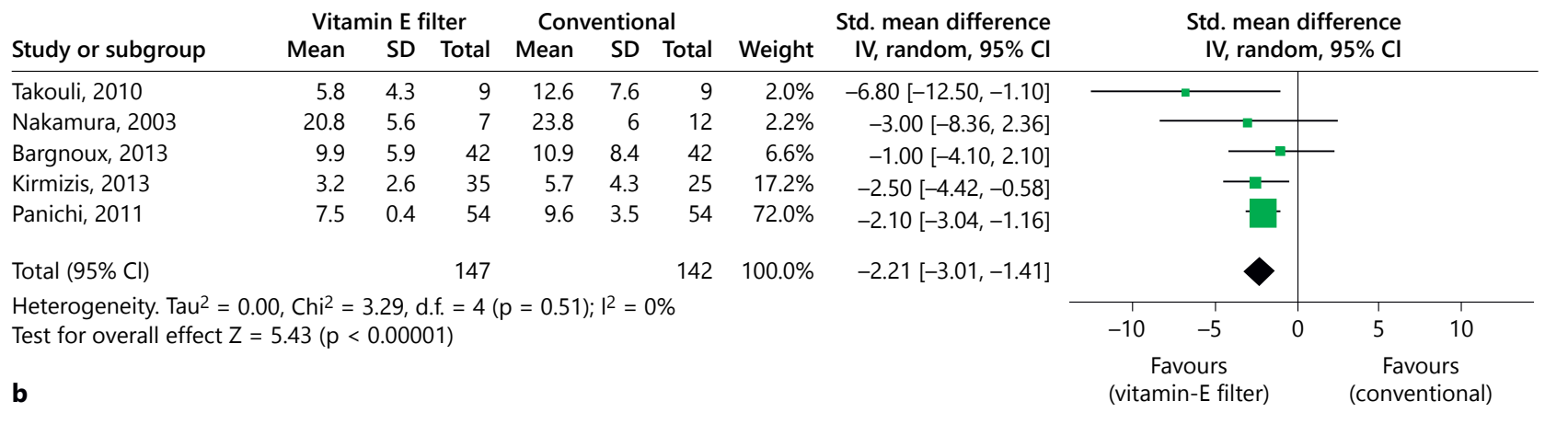

Fig. 9. Effects of ViE-m vs. conventional membrane on CRP (a) and IL-6 (b).

The same result was reported in one cross-over RCT [15]. Conversely, in 3 studies [23, 32, 61], IL-6 levels remained unaffected.

\section{Effects of ViE-m on Other Outcomes}

Lipid Profile

In data pooled from 13 studies $[11,28,31,48,50,51,58$, 64, 66-68, 70,71], ViE-m did not produce significant changes in total cholesterol levels ( 385 patients; MD $3.53 \mathrm{mg} / \mathrm{dl}$; $95 \%$ CI -2.98 to $10.04, \mathrm{chi}^{2}=20.11, \mathrm{p}=0.09 ; \mathrm{I}^{2}=35 \%$; fig. 10a).

Results from 2 other studies $[17,18]$ were in line with such findings.

ViE-m did not influence HDL cholesterol levels (9 studies, 233 patients; MD $0.22 \mathrm{mg} / \mathrm{dl} ; 95 \% \mathrm{CI}-1.47$ to $1.91 ; \mathrm{chi}^{2}=3.62, \mathrm{p}=0.89 ; \mathrm{I}^{2}=0 \%$; fig. $\left.10 \mathrm{~b}\right)[28,48,58,64$, $66-68,70,71]$, a finding that was concordant with those reported by 2 cross-over RCTs $[17,18]$, as well as LDL cholesterol levels ( 9 studies, 233 patients; MD $0.56 \mathrm{mg} / \mathrm{dl}$;
95\% CI -4.08 to $5.20 ; \mathrm{chi}^{2}=9.12, \mathrm{p}=0.33 ; \mathrm{I}^{2}=12 \%$; fig. 10c) $[28,48,58,64,66-68,70,71]$. Conversely, LDL and HDL cholesterol levels were decreased in both study groups in another cross-over non-RCT [43], while LDL cholesterol was significantly reduced by ViE-m in another cross-over RCT [18].

A meta-analysis of 10 studies [11, 31, 48, 51, 58, 64, 66, $68,70,71]$ indicated that ViE-m did not influence triglycerides levels (307 patients; MD $4.00 \mathrm{mg} / \mathrm{dl} ; 95 \% \mathrm{CI}-5.41$ to $13.41, \mathrm{chi}^{2}=2.99, \mathrm{p}=0.96 ; \mathrm{I}^{2}=0 \%$; fig. 11a). The same observation was reported also in other 4 studies $[17,18$, $28,43]$.

Pre- and Post-Dialysis Systolic and Diastolic Blood Pressure

Pre-dialysis systolic blood pressure (SBP) and diastolic blood pressure (DBP) were unchanged by ViE-m (4 studies; 161 patients; SBP: MD $1.59 \mathrm{~mm} \mathrm{Hg;} \mathrm{95 \%} \mathrm{CI}$ -1.41 to $4.58, \mathrm{chi}^{2}=2.09, \mathrm{p}=0.72 ; \mathrm{I}^{2}=0 \%$; fig. $11 \mathrm{~b}$; DBP: 
Total cholesterol

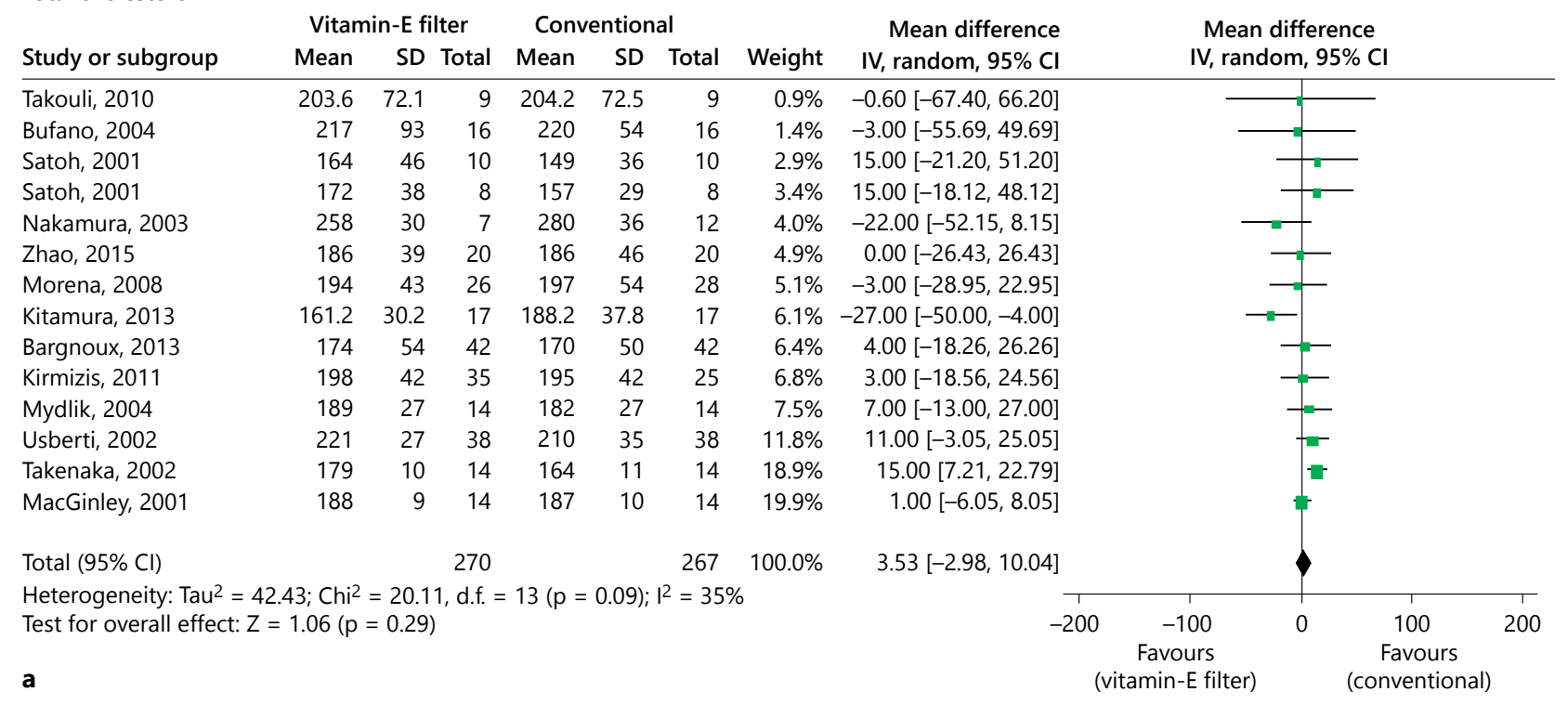

HDL cholesterol

\begin{tabular}{|c|c|c|c|c|c|c|c|}
\hline \multirow[b]{2}{*}{ Study or subgroup } & \multicolumn{3}{|c|}{ Vitamin-E filter } & \multicolumn{3}{|c|}{ Conventional } & \multirow[b]{2}{*}{ Weight } \\
\hline & Mean & SD & Total & Mean & SD & Total & \\
\hline Nakamura, 2003 & 40 & 15 & 7 & 40 & 16 & 12 & $1.4 \%$ \\
\hline Kitamura, 2013 & 38.2 & 14.3 & 17 & 46 & 19.9 & 17 & $2.1 \%$ \\
\hline Mydlik, 2004 & 50 & 15 & 14 & 46 & 12 & 14 & $2.8 \%$ \\
\hline Takouli, 2010 & 47.9 & 10.2 & 9 & 46.1 & 9.3 & 9 & $3.5 \%$ \\
\hline Zhao, 2015 & 39 & 12 & 20 & 39 & 12 & 20 & $5.2 \%$ \\
\hline Bufano, 2004 & 46 & 8 & 16 & 43 & 12 & 16 & $5.7 \%$ \\
\hline Takenaka, 2002 & 58 & 5 & 14 & 59 & 6 & 14 & $17.0 \%$ \\
\hline Kirmizis, 2011 & 41 & 6 & 35 & 40 & 8 & 25 & $20.7 \%$ \\
\hline MacGinley, 2001 & 43 & 4 & 14 & 43 & 3 & 14 & $41.6 \%$ \\
\hline Total $(95 \% \mathrm{Cl})$ & & & 146 & & & 141 & $100.0 \%$ \\
\hline
\end{tabular}

\section{b}

Mean difference
IV, random, 95\% Cl

LDL cholesterol

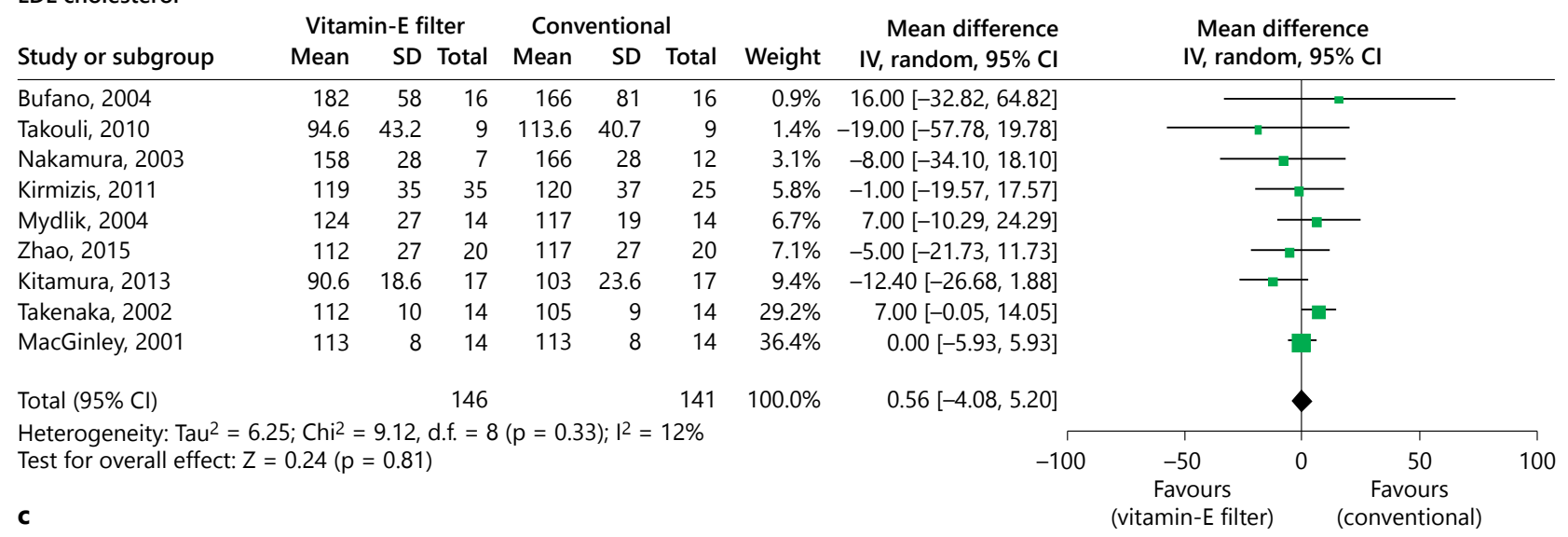

Fig. 10. Effects of ViE-m vs. conventional membrane on total (a), HDL and LDL cholesterol (b, c).

Vitamin-E vs. Conventional Dialysis: A Meta-Analysis
Blood Purif 2017;43:101-122

DOI: $10.1159 / 000453444$ 
MD $-0.64 \mathrm{~mm} \mathrm{Hg} ; 95 \% \mathrm{CI}-2.43$ to $1.16, \mathrm{chi}^{2}=1.95, \mathrm{p}=$ 0.75 ; $\mathrm{I}^{2}=0 \%$; fig. 11c) $[16,22,52,62]$.

These results were consistent with findings from another single study [32].

Similarly, in a meta-analysis of 3 studies, ViE-m had no effects on post-dialysis SBP and DBP (145 patients, SBP: MD $6.54 \mathrm{~mm} \mathrm{Hg}$; $95 \%$ CI -5.42 to $18.51, \mathrm{chi}^{2}=8.80$, $\mathrm{p}=0.03 ; \mathrm{I}^{2}=66 \%$; fig. 11d; DBP: MD $2.18 \mathrm{~mm} \mathrm{Hg} ; 95 \%$ CI -2.92 to $7.28, \mathrm{chi}^{2}=4.93, \mathrm{p}=0.18 ; \mathrm{I}^{2}=39 \%$; fig. $12 \mathrm{a}$ ). Findings from one additional RCT [32] were comparable with all these results.

\section{$\mathrm{Kt} / \mathrm{V}$}

A meta-analysis of 10 studies (180 patients) $[15,16,18$, $19,23,50,51,54,64,69$ ] showed that ViE-m did not influence $\mathrm{Kt} / \mathrm{V}$ (MD -0.05 ; $95 \% \mathrm{CI}-0.11$ to 0.02 ; fig. $12 \mathrm{~b}$ ). The high heterogeneity $\left(\mathrm{chi}^{2}=34.05, \mathrm{p}=0.0002 ; \mathrm{I}^{2}=\right.$ $71 \%$ ) was nullified by excluding data from 3 studies [ 15 , $16,64]$.

\section{Albumin}

Albumin levels were unaffected by ViE-m in data pooled from 8 studies $[11,16,22,50,51,64,69,70]$ (261 patients; MD $0.02 \mathrm{mg} / \mathrm{dl}$; $95 \%$ CI -0.08 to 0.12 ; fig. $12 \mathrm{c}$ ). This analysis had high heterogeneity $\left(\mathrm{chi}^{2}=37.49, \mathrm{p}<0.0001 ; \mathrm{I}^{2}=76 \%\right)$ that was almost fully explained by the only 2 studies with a parallel design $[69,70]\left(\mathrm{chi}^{2}=7.32, \mathrm{p}=0.40, \mathrm{I}^{2}=4 \%\right)$. Albumin remained unaffected in 2 additional RCTs $[17,32]$.

Uric Acid

ViE-m did not affect uric acid levels in a meta-analysis of 3 studies [49, 51, 64] (55 patients; MD $-0.26 \mathrm{mg} / \mathrm{dl}$; $95 \%$ CI -0.74 to 0.22 ; $\mathrm{chi}^{2}=3.26, \mathrm{p}=0.20 ; \mathrm{I}^{2}=39 \%$; fig. 13a). Results from another single study [58] were consistent with these findings.

\section{WBCs Count}

In data pooled from 3 studies $[59,64,70]$ (76 patients), ViE-m had no impact on the total WBC count (MD 0.02 count $/ \mathrm{mm}^{3} ; 95 \% \mathrm{CI}-0.87$ to $0.92 ; \mathrm{chi}^{2}=3.06, \mathrm{p}=$ 0.22 ; $\mathrm{I}^{2}=35 \%$; fig. $13 \mathrm{~b}$ ). These results were consistent with findings from another single study [20].

Conversely, in one cross-over RCT [18], WBC count was decreased in the 2 study groups.

\section{Publication Bias}

Visual inspection of the funnel plot and the Egger's regression test indicate that the presence of publication bias was unlikely for all the outcomes considered, with the exception of blood vitamin $\mathrm{E}(\mathrm{p}=0.006$; online suppl.fig. $1-6)$.

\section{Discussion}

The aim of this systematic review was to evaluate all possible benefits of using ViE-m in comparison with conventional membranes on oxidative stress, inflammation, anemia and a series of other parameters of clinical relevance for HD patients.

Findings obtained are consistent with a positive effect of ViE-m on oxidative stress and chronic inflammation biomarkers. Conversely, only partial effects were noticed on anemia control; these effects were mostly represented by changes on ERI and TSAT.

The rationale for ViE-m to decrease free radical damage during HD therapy is provided by the original demonstration that these functionalized membranes present on the blood surface of hollow fibers a redox-active form of $a$-tocopherol [5]. This key prerequisite satisfies the mechanistic interpretation of earliest findings of an improved lipid peroxidation in patients treated with these dialyser membranes $[36,72,73]$.Vitamin $\mathrm{E}$ ( $\alpha$-tocopherol) has unique properties in the panorama of phenolic antioxidants, representing the sole biologically relevant and specific free radical scavenger capable of preventing a peroxidating process in cooperation with a series of glutathione and thioredoxin-dependent peroxidises abundantly expressed in tissues and biological fluids [74]. In addition to providing protection against lipid peroxidation, this concerted activity between the radical scavenging activity of vitamin $\mathrm{E}$ and cellular peroxidases may also influence the lipid signaling of cells regulating the response to the lipoxygenase-dependent control of cell death pathways [75].

Findings from our meta-analysis demonstrate that patients on ViE-m had significant improvements in 3 very sensitive clinical biomarkers of oxidative stress, namely, MDA, vitamin E and TBARS [3, 76]. In agreement with our findings, Yang et al. [9] found a significant reduction in TBARS and MDA levels analyzed together. Our results also agree with the findings presented by Sosa et al. [8] for MDA levels but not for TBARS.

Yang et al. [9] showed that the use of ViE-m coated membrane decreased Ox-LDL levels. However, the authors meta-analyzed data only from 2 studies. In our meta-analysis, the sensitivity analysis performed by eliminating 3 parallel studies from the 7 initially was the only one that was considered, and it was in agreement with these results.

Finally, in accordance with our observations, also Yang et al. [9] did not evidence differences in other parameters of oxidative stress, such as TAS levels. 
Triglycerides

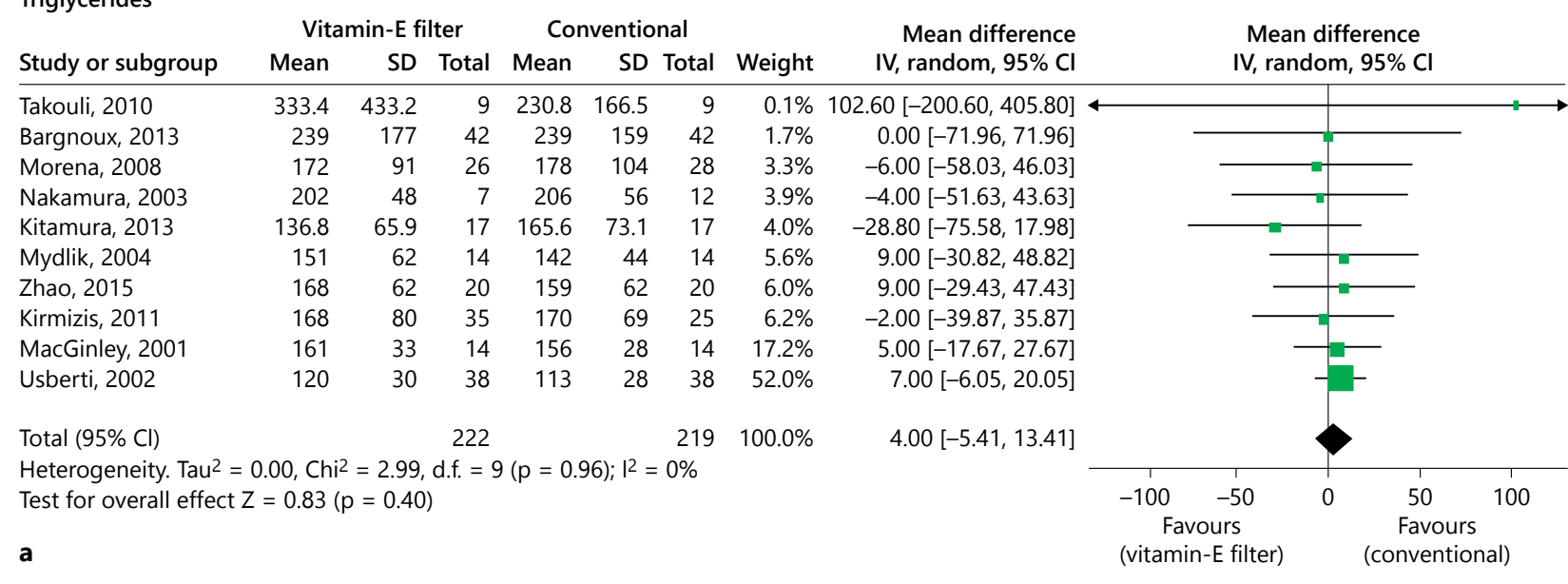

Predialysis systolic BP

\begin{tabular}{lrrrrrrr} 
& \multicolumn{3}{c}{ Vitamin-E filter } & \multicolumn{6}{c}{ Conventional } \\
Study or subgroup & Mean & SD & Total & Mean & SD & Total & Weight \\
\hline Matsumura, 2010 & 152 & 28 & 8 & 163 & 22 & 8 & $1.5 \%$ \\
Koremoto, 2012 & 157 & 27 & 28 & 156 & 31 & 28 & $3.9 \%$ \\
Koremoto, 2012 & 161 & 24 & 34 & 155 & 20 & 34 & $8.1 \%$ \\
Al-Jondeby, 2003 & 156 & 25 & 75 & 152 & 29 & 75 & $11.9 \%$ \\
Clermont, 2001 & 136 & 5 & 16 & 135 & 5 & 16 & $74.6 \%$
\end{tabular}

Total $(95 \% \mathrm{Cl}) \quad 161 \quad 161 \quad 100.0 \%$

Heterogeneity. Tau $^{2}=0.00, \mathrm{Chi}^{2}=2.09$, d.f. $=4(\mathrm{p}=0.72) ; \mathrm{I}^{2}=0 \%$

Test for overall effect: $Z=1.04(p=0.30)$

b

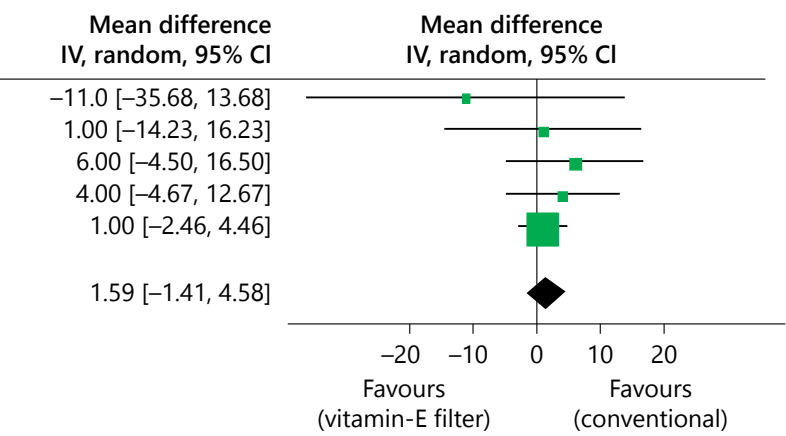

Predialysis diastolic BP

\begin{tabular}{lrrrrrrr} 
& \multicolumn{3}{c}{ Vitamin-E filter } & \multicolumn{2}{c}{ Conventional } \\
Study or subgroup & Mean & SD & Total & Mean & SD & Total & Weight \\
\hline Matsumura, 2010 & 75 & 15 & 8 & 82 & 13 & 8 & $1.7 \%$ \\
Koremoto, 2012 & 81 & 15 & 28 & 79 & 16 & 28 & $4.9 \%$ \\
Koremoto, 2012 & 78 & 17 & 34 & 76 & 12 & 34 & $6.6 \%$ \\
Al-Jondeby, 2003 & 82 & 16 & 75 & 82 & 16 & 75 & $12.3 \%$ \\
Clermont, 2001 & 72 & 3 & 16 & 73 & 3 & 16 & $74.5 \%$
\end{tabular}

$\begin{array}{r}\text { Mean difference } \\ \text { IV, random, 95\% Cl }\end{array}$
$-7.00[-20.75,6.75]$

Total $(95 \% \mathrm{Cl}) \quad 161 \quad 161 \quad 100.0 \%$

Heterogeneity. Tau $^{2}=0.00, \mathrm{Chi}^{2}=1.95$, d.f. $=4(\mathrm{p}=0.75) ; \mathrm{I}^{2}=0 \%$

Test for overall effect: $Z=0.69(p=0.49)$

C

Postdialysis systolic BP

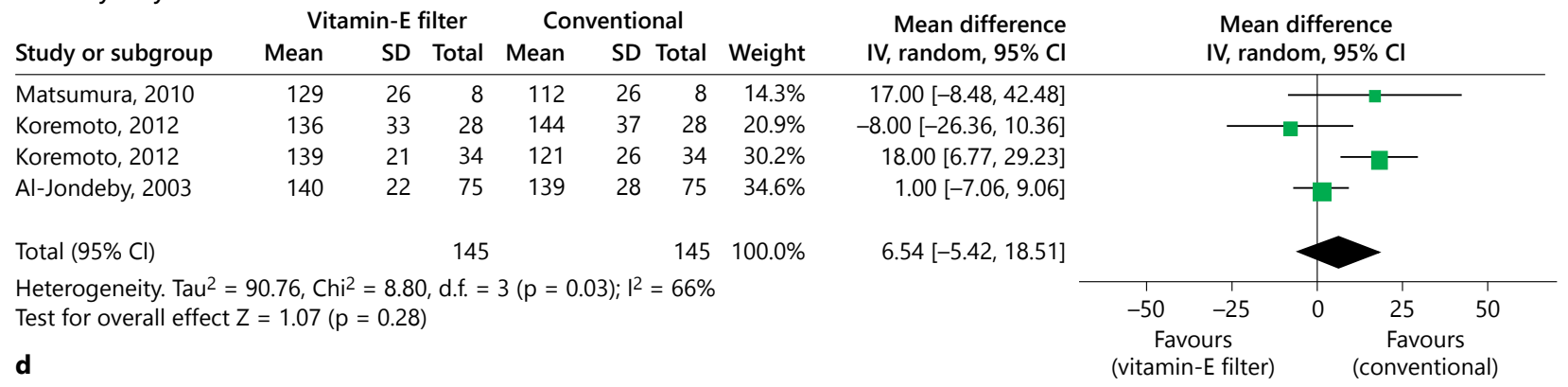

Fig. 11. Effects of ViE-m vs. conventional membrane on triglycerides (a), pre-dialysis SBP and DBP (b, c) and post-dialysis SBP (d).

Vitamin-E vs. Conventional Dialysis:

A Meta-Analysis
Blood Purif 2017;43:101-122

DOI: $10.1159 / 000453444$ 
Postdialysis diastolic BP

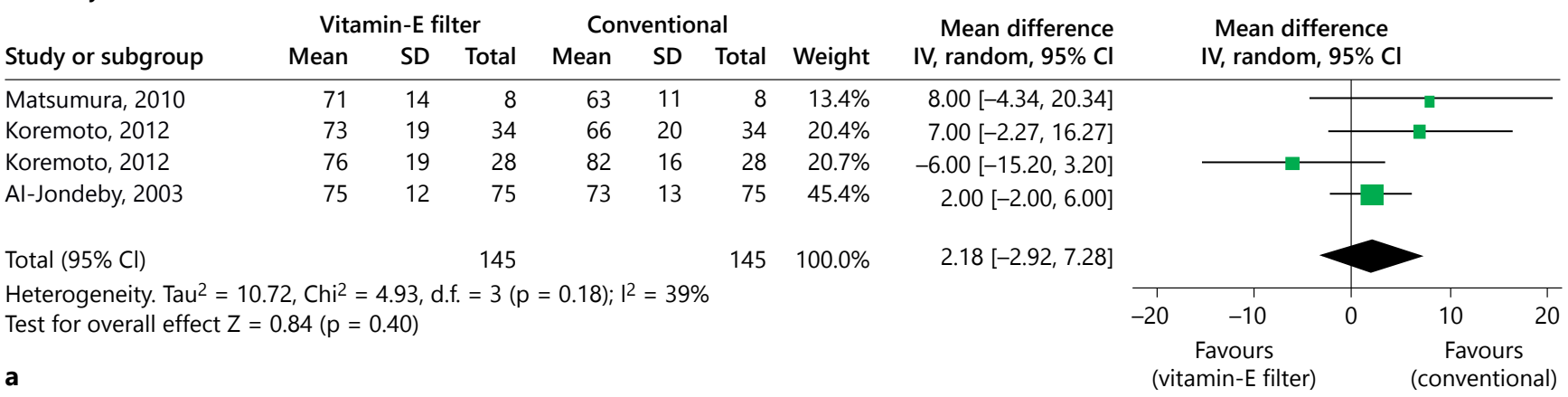

$\mathrm{Kt} / \mathrm{V}$

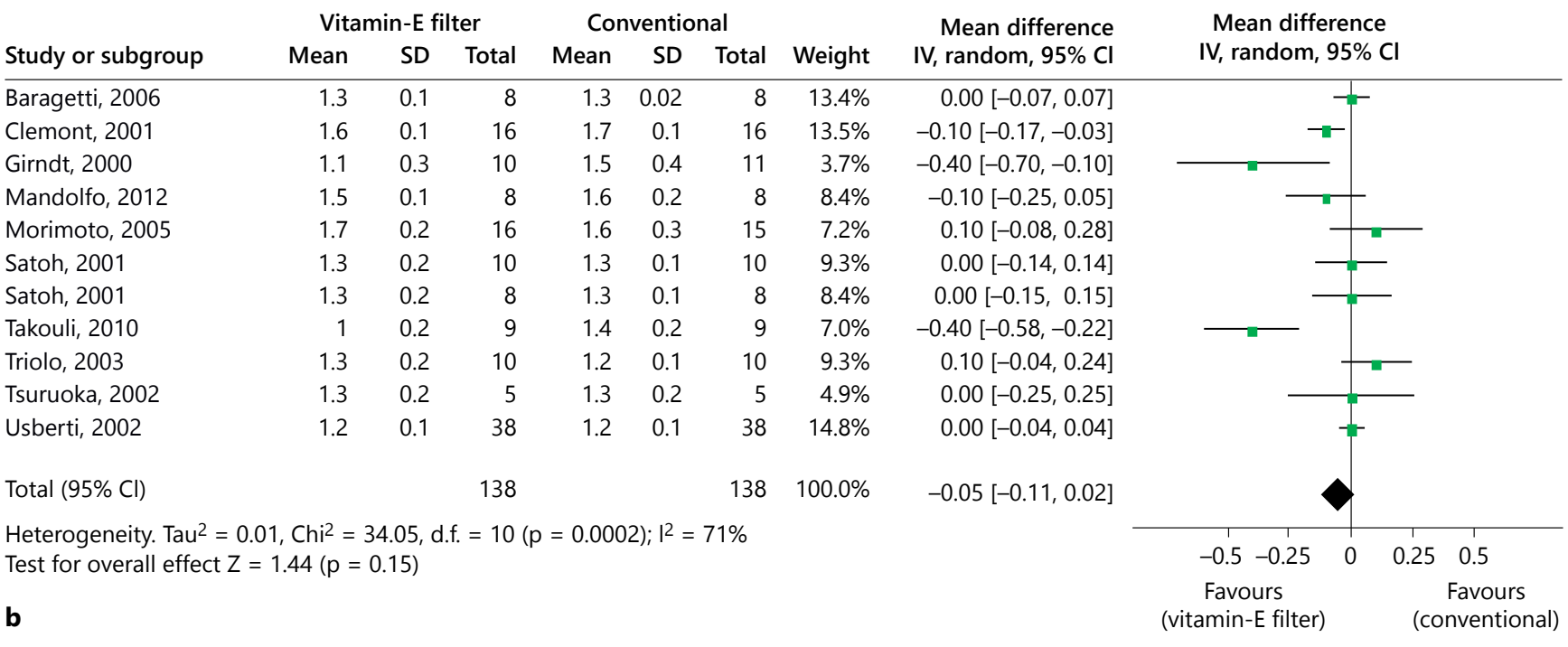

Albumin

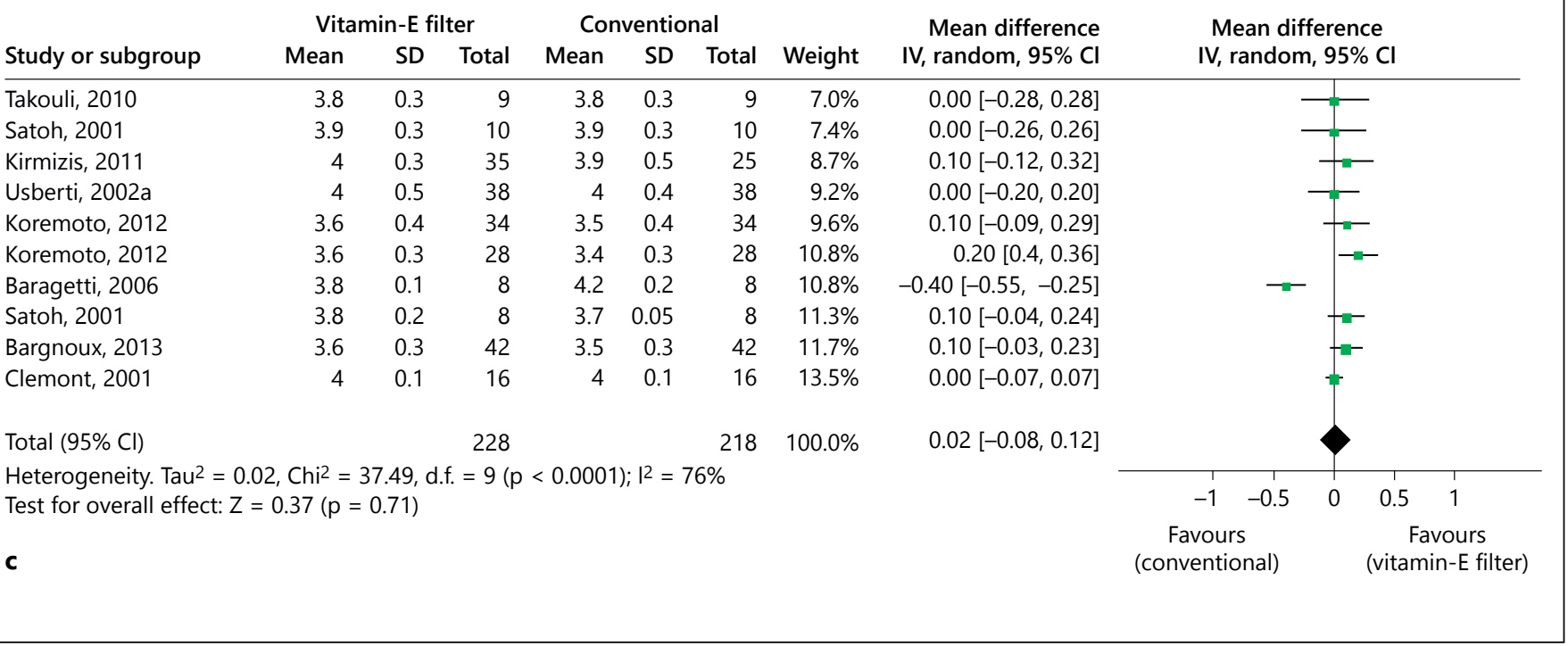

Fig. 12. Effects of ViE-m vs. conventional membrane on post-dialysis DBP (a), Kt/V (b) and albumin (c). 


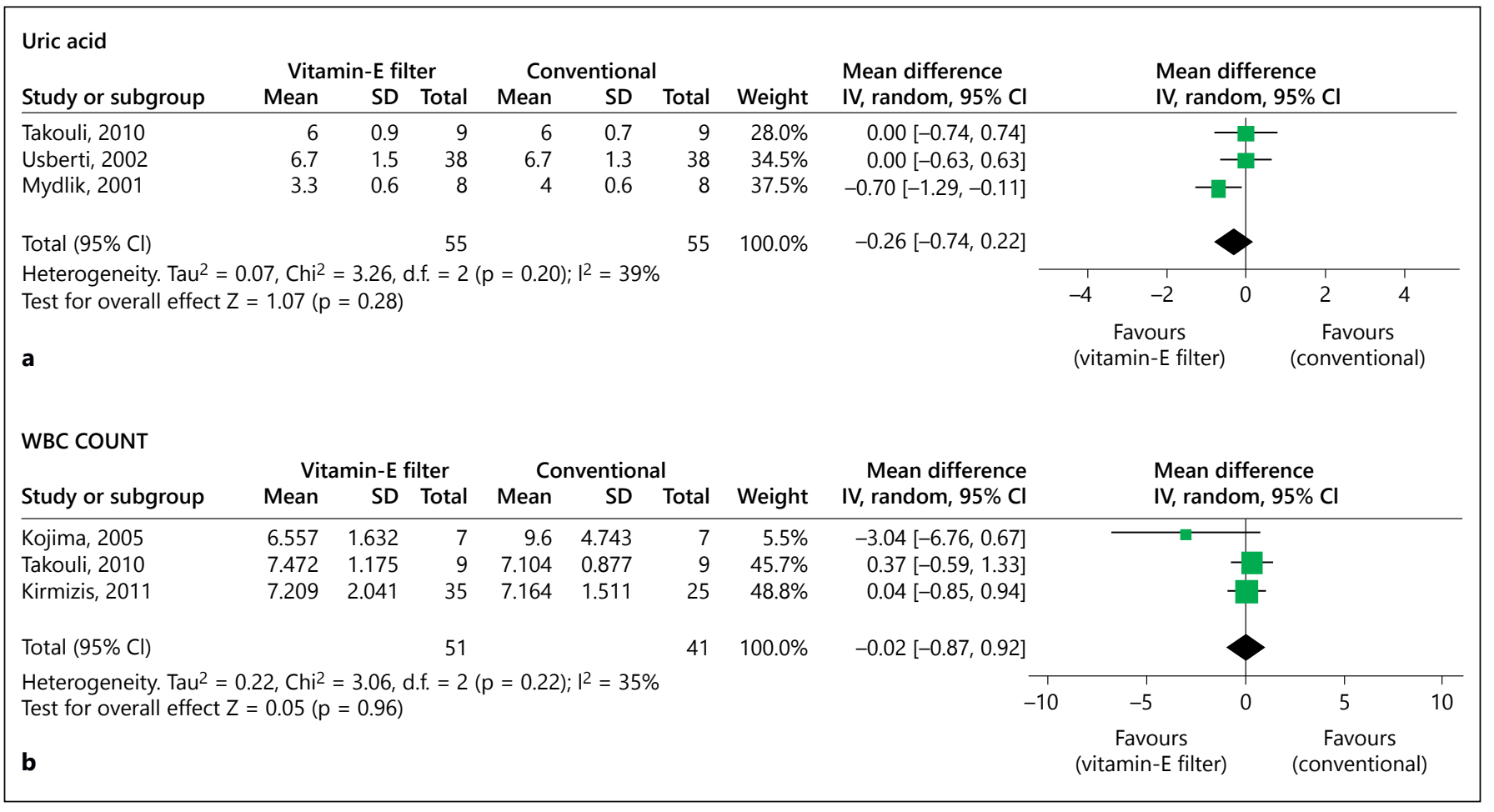

Fig. 13. Effects of ViE-m vs. conventional membrane on uric acid (a) and WBCs count (b).

The effect of ViE-m therapy on subclinical signs of inflammation was originally proposed to follow the same rational for the antioxidant and cytoprotective effects of these membranes discussed above [77]. This effect, originally proposed in a series of pilot clinical trials carried out on the cellulosic version of these functionalized membranes [77], has been confirmed in a series of recent studies on the synthetic (PS-PVP backbone) version of ViE-m as reviewed elsewhere [3]. Accordingly, a previous metaanalysis [9] demonstrated that the use of ViE-m significantly reduces IL-6 and CRP levels, hence improving the inflammatory status.

Conversely, in our analyses including a larger number of studies, a substantial improvement in inflammation status was confined only in the case of IL-6 biomarker.

Overall, these findings support the hypothesis that ViE-m may be endowed with anti-inflammatory and anti-oxidative effects that are worthy of further investigation in larger trials.

Early findings in literature [78] and more recent studies [79] suggest that ViE-m may help preserving vitamin E levels of circulating RBCs, a key requisite to provide resistance of these cells to a peroxidatic challenge and to prevent cell fragility and hemolysis or premature remov- al by the reticulo-endothelial system. In fact, patients on ViE-m have more chances of maintaining sufficient levels of a-tocopherol in their circulating erythrocytes, thus preventing the reduction in the RBC lifespan commonly observed in CKD patients [78].

One of the most interesting findings of our systematic review was that ViE-m reduces the ERI. This observation echoes previous findings based on fewer studies [7] and may have relevant clinical value, as ESA-resistance represents a major obstacle for anemia control in dialysis patients. Alternative therapeutic strategies for improving resistance to ESAs may be of foremost importance also for saving costs and improving other patient-centered outcomes.

Such finding on ERI is of interest; however, the general impact of ViE-m on anemia control remains inconclusive, as no significant benefits were noticed on total $\mathrm{Hb}$ levels and EPO dosages. The absence of effect on such parameters was in agreement with previous analyses focusing on fewer studies [7].

Finally, in line with previous meta-analyses [7], our results confirmed that the use of ViE-m did not influence other parameters of interest for dialysis patients, such as dialysis adequacy, lipid profile, intradialytic blood pres- 
sure, dialysis adequacy, serum albumin, uric acid and WBC count.

Our review has some points of strength and also a few limitations that deserve to be mentioned.

Strengths include a systematic search of medical databases, data extraction and analysis, and trial quality assessment by 2 independent reviewers based on current methodological standards. We also carefully analyzed the effect of ViE-m on a wide series of clinical parameters of interest, including but not limited to anemia, oxidative stress and inflammation, in order to frame the broadest possible clinical utility profile of these membranes.

Another strength of this review is the detailed exploration of the possible source of heterogeneity by thorough sensitivity analyses. Unfortunately, the overall quality of the included trials was low, since information about the random sequence generation, allocation concealment and blinding was lacking in a majority of them. In addition, our findings may be hampered by the small sample size and the short study duration of the included trials, which prevented the authors to analyze hard clinical endpoints such as fatal and non-fatal cardiovascular events, mortality and quality of life.

To conclude, to date, there is no substantial evidence supporting the utility of $\mathrm{ViE}-\mathrm{m}$ for ameliorating anemia, oxidative stress and inflammation in chronic HD patients, although the use of these membranes could be helpful for improving anti-anemic therapy.

Future trials adequately powered on hard, patientcentered, endpoints are advocated to clarify the potential role of ViE-m for improving the overall clinical management of chronic HD patients.

\section{Acknowledgments}

We thank Dr. Akiyama, Baragetti, Brancaccio, Calò, Gallieni, Gwinner, Odetti, Ronco, Sanaka and Senatore for providing full text articles upon request.

\section{Financial Disclosure}

The authors declare no conflict of interest with respect to the present work.

\section{Contributions}

Research idea and study design: F.G., D.B., G.D., R.B., and G.T., data acquisition: G.D., and R.B.; data analysis/interpretation: F.G., D.B., G.D., and R.B.; statistical analysis: D.B., G.D., R.B., and G.T.; manuscript writing: F.G., D.B., G.D., and R.B.

\section{References}

1 Krane V, Krieter DH, Olschewski M, Marz W, Mann JF, Ritz E, Wanner C: Dialyzer membrane characteristics and outcome of patients with type 2 diabetes on maintenance hemodialysis. Am J Kidney Dis 2007;49:267275 .

2 Boure T, Vanholder R: Which dialyser membrane to choose? Nephrol Dial Transplant 2004;19:293-296.

3 Piroddi M, Pilolli F, Aritomi M, Galli F: Vita$\min \mathrm{E}$ as a functional and biocompatibility modifier of synthetic hemodialyzer membranes: an overview of the literature on vitamin E-modified hemodialyzer membranes. Am J Nephrol 2012;35:559-572.

4 Galli F: Vitamin E-derived copolymers continue the challenge to hemodialysis biomaterials. World J Nephrol 2012;1:100-105.

5 Floridi A, Piroddi M, Pilolli F, Matsumoto Y, Aritomi M, Galli F: Analysis method and characterization of the antioxidant capacity of vitamin E-interactive polysulfone hemodialyzers. Acta Biomater 2009;5:2974-2982.

6 Morimoto H, Nakao K, Fukuoka K, Sarai A, Yano A, Kihara T, Fukuda S, Wada J, Makino $\mathrm{H}$ : Long-term use of vitamin E-coated polysulfone membrane reduces oxidative stress markers in haemodialysis patients. Nephrol Dial Transplant 2005;20:2775-2782.

7 Huang J, Yi B, Li AM, Zhang H: Effects of vitamin E-coated dialysis membranes on anemia, nutrition and dyslipidemia status in hemodialysis patients: a meta-analysis. Ren Fail 2015;37:398-407.

8 Sosa MA, Balk EM, Lau J, Liangos O, Balakrishnan VS, Madias NE, Pereira BJ, Jaber BL: A systematic review of the effect of the excebrane dialyser on biomarkers of lipid peroxidation. Nephrol Dial Transplant 2006;21: 2825-2833.

9 Yang SK, Xiao L, Xu B, Xu XX, Liu FY, Sun L: Effects of vitamin E-coated dialyzer on oxidative stress and inflammation status in hemodialysis patients: a systematic review and meta-analysis. Ren Fail 2014;36:722-731.

10 Usberti M, Gerardi G, Bufano G, Tira P, Micheli A, Albertini A, Floridi A, Di Lorenzo D, Galli F: Effects of erythropoietin and vitamin E-modified membrane on plasma oxidative stress markers and anemia of hemodialyzed patients. Am J Kidney Dis 2002;40: 590-599.

11 Bargnoux AS, Cristol JP, Jaussent I, Chalabi L, Bories P, Dion JJ, Henri P, Delage M, Dupuy
AM, Badiou S, Canaud B, Morena M: Vitamin E-coated polysulfone membrane improved red blood cell antioxidant status in hemodialysis patients. J Nephrol 2013;26:556-563.

12 Hozo SP, Djulbegovic B, Hozo I: Estimating the mean and variance from the median, range, and the size of a sample. BMC Med Res Methodol 2005;5:13.

13 Higgins JP, Thompson SG, Deeks JJ, Altman DG: Measuring inconsistency in meta-analyses. BMJ 2003;327:557-560.

14 Schiecke G, Gwinner W, Radermacher J, Bahlmann J, Lonnemann G: Long-term effects of vitamin E-bonded dialysis membrane on mononuclear cell activation, malondialdehyde generation and endothelial function in ESRD patients. Contrib Nephrol 1999;127:243-250.

15 Girndt M, Lengler S, Kaul H, Sester U, Sester $\mathrm{M}$, Kohler H: Prospective crossover trial of the influence of vitamin E-coated dialyzer membranes on T-cell activation and cytokine induction. Am J Kidney Dis 2000;35:95-104.

16 Clermont G, Lecour S, Cabanne JF, Motte G, Guilland JC, Chevet D, Rochette L: Vitamin E-coated dialyzer reduces oxidative stress in hemodialysis patients. Free Radic Biol Med 2001;31:233-241. 
17 Senatore M, Nicoletti A, Rizzuto G: Is the bioreactivity of vitamin-E-modified dialyzer an expression of increased plasmatic vitamin $\mathrm{E}$ concentration? Nephron 2002;92:487-489.

18 Tsuruoka S, Kawaguchi A, Nishiki K, Hayasaka T, Fukushima C, Sugimoto K, Saito T, Fujimura A: Vitamin E-bonded hemodialyzer improves neutrophil function and oxidative stress in patients with end-stage renal failure. Am J Kidney Dis 2002;39:127-133.

19 Morimoto H, Nakao K, Fukuoka K, Sarai A, Yano A, Kihara T, Fukuda S, Wada J, Makino $\mathrm{H}$ : Long-term use of vitamin E-coated polysulfone membrane reduces oxidative stress markers in haemodialysis patients. Nephrol Dial Transplant 2005;20:2775-2782.

20 Sato M, Morita H, Ema H, Yamaguchi S, Amano I: Effect of different dialyzer membranes on cutaneous microcirculation during hemodialysis. Clin Nephrol 2006;66:426-432.

21 Panichi V, Rosati A, Paoletti S, Ferrandello P, Migliori M, Beati S, Bernabini G, Daini R, Casani A, Angelini D, Parrini M, Rossi A, Petrone I, Barsotti G, Donadio C, Donati G, Grazi G, Manca Rizza G, Garosi G, Sansoni E, Braccagni B, Sidoti A, Boracelli D, Biagioli M, Moriconi L, Finato V, Mannarino A, Grimaldi C, Pansa F, Imperiali P, Mura C, Bianchi S, Bigazzi R: A vitamin E-coated polysulfone membrane reduces serum levels of inflammatory markers and resistance to erythropoietin-stimulating agents in hemodialysis patients: results of a randomized cross-over multicenter trial. Blood Purif 2011;32:7-14.

22 Koremoto $M$, Takahara N, Takahashi $M$, Okada Y, Satoh K, Kimura T, Hirai T, Ebihara I, Nagasaku D, Miyata S, Maniwa S, Kouzuma T, Arimura T, Kamei J: Improvement of intradialytic hypotension in diabetic hemodialysis patients using vitamin E-bonded polysulfone membrane dialyzers. Artif Organs 2012;36:901-910

23 Mandolfo S, Corradi B, Bucci R, Farina M, Pilolli F, Galli F: Evaluation of the impact of a new synthetic vitamin E-bonded membrane on anemia and rHuEPO requirement in ESRD patients with central venous catheters: a pilot study. Int Urol Nephrol 2012;44:14931500.

24 Mune M, Yukawa S, Kishino M, Otani H, Kimura K, Nishikawa O, Takahashi T, Kodama N, Saika Y, Yamada Y: Effect of vitamin $\mathrm{E}$ on lipid metabolism and atherosclerosis in ESRD patients. Kidney Int Suppl 1999; 71:S126-S129.

25 Shimazu T, Kondo S, Toyama K, Komurai M, Ohminato M, Yasuda T, Sato T, Maeba T, Maruyama H, Owada S, Ishida M: Effect of vitamin E-modified regenerative cellulose membrane on neutrophil superoxide anion radical production and lipid peroxidation. Contrib Nephrol 1999;127:251-260.

26 Eiselt J, Racek J, Trefil L, Opatrný K Jr: Effects of a vitamin E-modified dialysis membrane and vitamin $\mathrm{C}$ infusion on oxidative stress in hemodialysis patients. Artif Organs 2001;25: 430-436.
27 Kobayashi S, Moriya H, Aso K, Ohtake T: Vitamin E-bonded hemodialyzer improves atherosclerosis associated with a rheological improvement of circulating red blood cells. Kidney Int 2003;63:1881-1887.

28 Bufano G, Usberti M, Mandolfo S, Malberti F Piroddi M, Galli F: Von Willebrand factor and autoantibodies against oxidized LDL in hemodialysis patients treated with vitamin Emodified dialyzers. Int J Artif Organs 2004;27: 214-221.

29 Zhao Z, Shi WZ: Clinical study of anti-oxidant effects of vitamin E-modified membrane on hemodialysis patients. Chin J Pract Int Med 2004;24:479-481.

30 Yang CC, Hsu SP, Wu MS, Hsu SM, Chien CT: Effects of vitamin C infusion and vitamin E-coated membrane on hemodialysis-induced oxidative stress. Kidney Int 2006;69: 706-714.

31 Morena M, Gausson V, Mothu N, Bouchet JL, Chanas M, Grandvuillemin M, Robert A, Vela C, Canaud B, Cristol JP, Olmer M: Regional variations of low-density lipoprotein oxidizability in hemodialysis patients may explain discrepancies in interventional therapy on oxidative profile. Blood Purif 2008;26:300310.

32 Andrulli S, Di Filippo S, Manzoni C, Stefanelli L, Floridi A, Galli F, Locatelli F: Effect of synthetic vitamin E-bonded membrane on responsiveness to erythropoiesis-stimulating agents in hemodialysis patients: a pilot study. Nephron Clin Pract 2010;115:c82-c89.

33 Wang J, Liu S, Li X, Qi H, Zhuang S, Yan H: Effects of different dialyzers on oxidative stress for maintenance hemodialysis patients. Shanghai Med J 2012;35:601-604.

34 Sanaka T, Mochizuki T, Kinugasa E, Kusano E, Ohwada S, Kuno T, Kojima K, Kobayashi S, Satoh M, Shimada N, Nakao K, Nakazawa $R$, Nishimura $H$, Noiri E, Shigematsu T, Tomo T, Maeda T; VEESA Study Group: Randomized controlled open-label trial of vitamin E-bonded polysulfone dialyzer and erythropoiesis-stimulating agent response. Clin J Am Soc Nephrol 2013;8:969-978.

35 Lines SW, Carter AM, Dunn EJ, Lindley EJ, Tattersall JE, Wright MJ: A randomized controlled trial evaluating the erythropoiesis stimulating agent sparing potential of a vitamin E-bonded polysulfone dialysis membrane. Nephrol Dial Transplant 2014;29:649656.

36 Buoncristiani U, Galli F, Rovidati S, Albertini MC, Campus G, Canestrari F: Oxidative damage during hemodialysis using a vitamin-Emodified dialysis membrane: a preliminary characterization. Nephron 1997;77:57-61.

37 Galli F, Rovidati S, Chiarantini L, Campus G, Canestrari F, Buoncristiani U: Bioreactivity and biocompatibility of a vitamin E-modified multi-layer hemodialysis filter. Kidney Int 1998; 54:580-589.

38 Galli F, Rovidati S, Benedetti S, Canestrari F, Ferraro B, Floridi A, Buoncristiani U: Lipid peroxidation, leukocyte function and apopto- sis in hemodialysis patients treated with vitamin E-modified filters. Contrib Nephrol 1999;127:156-171.

39 Odetti P, Robaudo C, Valentini S, Gurreri G, Garibaldi S, Angeletti S, Deferrari G: Effect of a new vitamin E-coated membrane on glycoxidation during hemodialysis. Contrib Nephrol 1999;127:192-199.

40 Racek J, Eiselt J, Trefil L, Opatrny KJ: Influence of vitamin $\mathrm{C}$ infusion and vitamin $\mathrm{E}$ modified membrane on markers of oxidative stress during hemodialysis. Nephrol Dial Transplant 1999;14:A165.

41 Sommerburg, Sostmann K, Grune T, Ehrich $\mathrm{JH}$ : Oxidative stress in hemodialysis patients treated with a dialysis membrane which has alpha-tocopherol bonded to its surface. Biofactors 1999;10:121-124.

42 Taccone-Gallucci M, Meloni C, Lubrano R, Morosetti M, Palombo G, Cianciulli P, Scoppi P, Castello MA, Casciani CU: Chronic haemolysis and erythrocyte survival in haemodialysis patients treated with vitamin E-modified dialysis filters. Contrib Nephrol 1999; 127:44-48.

43 Tanaka H, Nishikawa O, Yukawa S, Yoshimoto $\mathrm{M}$, Nishide I: [Effects of hemodialysis membrane on serum lipid profile of maintenance hemodialysis patients]. Nihon Jinzo Gakkai Shi 1999;41:1-7.

44 Bonnefont-Rousselot D, Lehmann E, Jaudon MC, Delattre J, Perrone B, Rechke JP: Blood oxidative stress and lipoprotein oxidizability in haemodialysis patients: effect of the use of a vitamin E-coated dialysis membrane. Nephrol Dial Transplant 2000;15:2020-2028.

45 Huraib S, Tanimu D, Shaheen F, Hejaili F, Giles C, Pagayon V: Effect of vitamin-E-modified dialysers on dialyser clotting, erythropoietin and heparin dosage: a comparative crossover study. Am J Nephrol 2000;20:364368

46 Miyazaki $\mathrm{H}$, Matsuoka $\mathrm{H}$, Itabe $\mathrm{H}$, Usui $\mathrm{M}$, Ueda S, Okuda S, Imaizumi T: Hemodialysis impairs endothelial function via oxidative stress: effects of vitamin E-coated dialyzer. Circulation 2000;101:1002-1006.

47 Galli F, Varga Z, Balla J, Ferraro B, Canestrari F, Floridi A, Kakuk G, Buoncristiani U: Vitamin E, lipid profile, and peroxidation in hemodialysis patients. Kidney Int Suppl 2001; 78:S148-S154.

48 MacGinley R, Westhuyzen J, Saltissi D, Morgan C, Healy H, Thirlwell GK, Disney AP: Evaluation of a novel vitamin E coated cellulosic membrane hollow fiber dialyzer. Asaio J 2001;47:66-73.

49 Mydlik M, Derzsiova K, Racz O, Sipulova A, Lovasova E, Petrovicova J: A modified dialyzer with vitamin $\mathrm{E}$ and antioxidant defense parameters. Kidney Int Suppl 2001;78:S144S147.

50 Satoh M, Yamasaki Y, Nagake Y, Kasahara J, Hashimoto M, Nakanishi N, Makino H: Oxidative stress is reduced by the long-term use of vitamin E-coated dialysis filters. Kidney Int 2001;59:1943-1950. 
51 Usberti M, Gerardi G, Micheli A, Tira P, Bufano G, Gaggia P, Movilli E, Cancarini GC, De Marinis S, D'Avolio G, Broccoli R, Manganoni A, Albertin A, Di Lorenzo D: Effects of a vitamin E-bonded membrane and of glutathione on anemia and erythropoietin requirements in hemodialysis patients. J Nephrol 2002; 15:558-564.

52 Al-Jondeby MS, Cabaguing IT, Pajarillo AA, Hawas FA, Mousa DH, Al-Sulaiman MH, Shaheen FA, Al-Khader AA: Comparative crossover controlled study using polysulphone and vitamin E coated dialyzers. Saudi Med J 2003;24:265-268.

53 Nakatan T, Takemoto Y, Tsuchida AK: The effect of vitamin E-bonded dialyzer membrane on red blood cell survival in hemodialyzed patients. Artif Organs 2003;27:214-217.

54 Triolo L, Malaguti M, Ansali F, Comunian MC, Arcangeloni O, Coppolino F, Marrocco F, Sicoli R, Biagini M: Vitamin E-bonded cellulose membrane, lipoperoxidation, and anemia in hemodialysis patients. Artif Cells Blood Substit Immobil Biotechnol 2003;31: 185-191.

55 Westhuyzen J, Saltissi D, Stanbury V: Oxidative stress and erythrocyte integrity in endstage renal failure patients hemodialysed using a vitamin E-modified membrane. Ann Clin Lab Sci 2003;33:3-10.

56 Hara T, Takahashi N, Kiyomoto H, Aki Y, Fujioka H, Shokoji T, Matsubara K, Moriwaki K, Kondo N, Kiyomoto K, Hirohata M, Ishizu T, Akiyama K, Nishiyama A, Ohmori K, Kohno M: Reduction of oxidized lowdensity lipoprotein by the long-term use of vitamin E-coated dialyzers in hemodialysis patients. Dial Transplant 2004;33:197207.

57 Libetta C, Zucchi M, Gori E, Sepe V, Galli F, Meloni F, Milanesi F, Dal Canton A: Vitamin E-loaded dialyzer resets PBMC-operated cytokine network in dialysis patients. Kidney Int 2004;65:1473-1480.

58 Mydlík M, Derzsiová K, Rácz O, Sipulová A, Lovásová E, Molcányiová A, Petrovicová J: Vitamin E-coated dialyzer and antioxidant defense parameters: three-month study. Semin Nephrol 2004;24:525-531.

59 Kojima K, Oda K, Homma H, Takahashi K, Kanda Y, Inokami T, Uchida S: Effect of vitamin E-bonded dialyzer on eosinophilia in haemodialysis patients. Nephrol Dial Transplant 2005;20:1932-1935.
60 Cruz DN, De Cal M, Garzotto F, Brendolan A, Nalesso D, Corradi V, Ronco C: Effect of vitamin E-coated dialysis membranes on anemia in patients with chronic kidney disease: an Italian multicenter study. Int J Artif Organs 2008;31:545-552.

61 Aoun B, Janssen-Lozinska Y, Ulinski T: Effect of vitamin $\mathrm{E}$ coated dialyzers on anticoagulation requirement in hemodialyzed children. Saudi J Kidney Dis Transpl 2010;21: 466-470.

62 Matsumura M, Sasaki H, Sekizuka K, Sano H, Ogawa K, Shimizu C, Yoshida H, Kobayashi S, Koremoto M, Aritomi M, Ueki K: Improved management of intradialytic hypotension (IDH) using vitamin E-bonded polysulfone membrane dialyzer. Int J Artif Organs 2010;33:147-153.

63 Sarandol E, Dirican M, Ocak N, Serdar Z Sonmezisik F, Dilek K: The effects of vitamin E-coated dialysis membranes on serum paraoxonase activity in hemodialysis patients. J Nephrol 2010;23:431-437.

64 Takouli L, Hadjiyannakos D, Metaxaki P, Sideris V, Filiopoulos V, Anogiati A, Vlassopoulos D: Vitamin E-coated cellulose acetate dialysis membrane: long-term effect on inflammation and oxidative stress. Ren Fail 2010;32: 287-293.

65 Calo LA, Naso A, D’Angelo A, Pagnin E, Zanardo M, Puato M, Rebeschini M, Landini S, Feriani M, Perego A, Malagoli A, Zagatti R, Calzavara P, Cascone C, Davis PA: Molecular biology-based assessment of vitamin E-coated dialyzer effects on oxidative stress, inflammation, and vascular remodeling. Artif Organs 2011;35:E33-E39.

66 Kitamura Y, Kamimura K, Yoshioka N, Hosotani Y, Tsuchida K, Koremoto M, Minakuchi J: The effect of vitamin E-bonded polysulfone membrane dialyzer on a new oxidative lipid marker. J Artif Organs 2013;16:206-210.

67 Takenaka T, Takahashi K, Kobayashi T, Oshima E, Iwasaki S, Suzuki H: Oxidized low density lipoprotein (Ox-LDL) as a marker of atherosclerosis in hemodialysis (HD) patients. Clin Nephrol 2002;58:33-37.

68 Nakamura T, Kawagoe Y, Matsuda T, Takahashi Y, Sekizuka K, Ebihara I, Koide H: Effects of LDL apheresis and vitamin E-modified membrane on carotid atherosclerosis in hemodialyzed patients with arteriosclerosis obliterans. Kidney Blood Press Res 2003;26: 185-191.
69 Baragetti I, Furiani S, Vettoretti S, Raselli S, Maggi FM, Galli F, Catapano AL, Buccianti G: Role of vitamin E-coated membrane in reducing advanced glycation end products in hemodialysis patients: a pilot study. Blood Purif 2006;24:369-376.

70 Kirmizis D, Papagianni A, Belechri AM, Memmos D: Effects of vitamin E-coated membrane dialyser on markers of oxidative stress and inflammation in patients on chronic haemodialysis. Nephrol Dial Transplant 2011;26:2296-2301.

71 Zhao YW, Shi ZW: Effects of vitamin E-modified dialysis membrane on lipids in patients on hemodialysis. Archives of Biological Sciences 2015;67:503-509.

72 Galli F, Rovidati S, Chiarantini L, Campus G, Canestrari F, Buoncristiani U: Bioreactivity and biocompatibility of a vitamin E-modified multi-layer hemodialysis filter. Kidney Int 1998;54:580-589.

73 Galli F, Rovidati S, Benedetti S, Canestrari F, Ferraro B, Floridi A, Buoncristiani U: Lipid peroxidation, leukocyte function and apoptosis in hemodialysis patients treated with vitamin E-modified filters. Contrib Nephrol 1999;127:156-171.

74 Forman HJ, Davies KJ, Ursini F: How do nutritional antioxidants really work: nucleophilic tone and para-hormesis versus free radical scavenging in vivo. Free Radic Biol Med 2014; 66:24-35.

75 Galli F, Azzi A, Birringer M, Cook-Mills JM, Eggersdorfer M, Frank J, Lorkowski S, Ozer NK: Vitamin E: emerging aspects and new directions. Free Radic Biol Med 2016;pii:S08915849(16)30432-4.

76 Galli F, Piroddi M, Annetti C, Aisa C, Floridi E, Floridi A: Oxidative stress and reactive oxygen species. Contrib Nephrol 2005;149:240-260.

77 Galli F, Ronco C: Oxidant stress in hemodialysis. Nephron 2000;84:1-5.

78 Usberti M, Gerardi G, Bufano G, Tira P, Micheli A, Albertini A, Floridi A, Di Lorenzo D, Galli F: Effects of erythropoietin and vitamin E-modified membrane on plasma oxidative stress markers and anemia of hemodialyzed patients. Am J Kidney Dis 2002;40:590-599.

79 Bargnoux AS, Cristol JP, Jaussent I, Chalabi L, Bories P, Dion JJ, Henri P, Delage M, Dupuy AM, Badiou S, Canaud B, Morena M: Vitamin E-coated polysulfone membrane improved red blood cell antioxidant status in hemodialysis patients. J Nephrol 2013;26:556-563. 